Astrophysical Journal

Preprint typeset using $\mathrm{LAT}_{\mathrm{E}} \mathrm{X}$ style emulateapj v. 5/2/11

\title{
THEORETICAL LIMITS ON MAGNETIC FIELD STRENGTHS IN LOW-MASS STARS
}

\author{
Matthew K. Browning ${ }^{1}$, Maria A. Weber ${ }^{1}$, Gilles Chabrier ${ }^{1,2}$, Angela P. Massey ${ }^{3}$ \\ Received Sept 7, 2015 ; Accepted Dec 15, 2015
}

\begin{abstract}
Observations have suggested that some low-mass stars have larger radii than predicted by 1-D structure models. Some theoretical models have invoked very strong interior magnetic fields (of order $1 \mathrm{MG}$ or more) as a possible cause of such large radii. Whether fields of that strength could in principle by generated by dynamo action in these objects is unclear, and we do not address the matter directly. Instead, we examine whether such fields could remain in the interior of a low-mass object for a significant time, and whether they would have any other obvious signatures. First, we estimate timescales for the loss of strong fields by magnetic buoyancy instabilities. We consider a range of field strengths and simple morphologies, including both idealized flux tubes and smooth layers of field. We confirm some of our analytical estimates using thin flux tube magnetohydrodynamic (MHD) simulations of the rise of buoyant fields in a fully-convective M-dwarf. Separately, we consider the Ohmic dissipation of such fields. We find that dissipation provides a complementary constraint to buoyancy: while small-scale, fibril fields might be regenerated faster than they rise, the dissipative heating associated with such fields would in some cases greatly exceed the luminosity of the star. We show how these constraints combine to yield limits on the internal field strength and morphology in low-mass stars. In particular, we find that for stars of 0.3 solar masses, no fields in flux tubes stronger than about $800 \mathrm{kG}$ are simultaneously consistent with both constraints.
\end{abstract}

Subject headings: convection, MHD, turbulence, stars: magnetic fields, stars: low-mass, brown dwarfs

\section{INTRODUCTION}

Low-mass stars are magnetic. In many cases that magnetism is observed at the stellar surface, either through proxies like chromospheric activity or coronal emission (e.g., Noyes et al. 1984; Pizzolato et al. 2003; Wright et al. 2011), through direct photometric monitoring of starspots (e.g. Basri et al. 2013; McQuillan et al. 2014), or through measurements of Zeeman broadening in magnetically sensitive lines or molecular bands (e.g., Reiners \& Basri 2007). Stellar flares of remarkable strength are also commonplace in low-mass stars (e.g., Osten et al. 2010) and are similarly believed to be linked to the presence and properties of a strong magnetic field.

The magnetism of these stars may influence their structure and evolution in a variety of ways. One of the most remarkable clues that this might be occurring has come from studies of the radii of active, low mass stars: in eclipsing K-dwarf and M-dwarf binaries, measured radii exceed model predictions in some cases by about 10 per cent (e.g., Torres \& Ribas 2002; López-Morales 2007; Morales et al. 2008; Stassun et al. 2012; Torres 2013). Most of the systems for which precise radius measurements are available are in close eclipsing binaries, which are expected to be rapidly rotating and strongly magnetic, though discrepancies also appear to be present in at least one longer-period system (Irwin et al. 2011). Several authors have attributed these seemingly anomalous radii and temperatures to the presence of remarkably strong interior magnetic fields.

\footnotetext{
${ }^{1}$ Dept of Physics and Astronomy, Stocker Road, University of Exeter, EX4 4QL, browning@astro.ex.ac.uk

2 Ecole Normale Superieure de Lyon, CRAL, UMR CNRS 5574, F-69364 Lyon Cedex 07, France

3 Department of Astronomy, Boston University, 725 Commonwealth Avenue, Boston, MA 02215, USA
}

Mullan \& MacDonald (2001), for example, argued that some of the observed properties of these stars could be explained if the interior was not fully convective but instead possessed a magnetic field strong enough to render the innermost parts of the star stable to convection. Using the stability analysis of Gough \& Tayler (1966), they estimated the required field strengths to be as great as $100 \mathrm{MG}$. Many other works have argued that less extreme magnetic fields might still lead to noticeable changes in the stellar radius, either through inhibition of convective transport or through the effects of cool starspots (e.g., Spruit \& Weiss 1986; Chabrier et al. 2007; MacDonald \& Mullan 2012, 2013, 2014, 2015, hereafter collectively referred to as MM). Very recently, Feiden \& Chabover (2012, 2013, 2014, hereafter FC14) have presented stellar models using a modified version of the Dartmouth stellar evolution code that attempts to account for the effects of magnetism within the context of mixing-length theory; they concluded that only fields with strengths greater than $10 \mathrm{MG}$ in the deep interior would be able to modify convection enough to explain the observed radii of fully convective stars (though, as noted below, they ultimately found such fields untenable).

Whether fields of such strength could in reality be generated in a star, or even just maintained if somehow produced, is not entirely clear. In general the fields are believed to arise from the action of a magnetic dynamo - a process that converts kinetic energy to magnetic (see, e.g., Moffatt 1978). The maximum field strengths achievable in principle by dynamo action in any given instance are not easy to estimate: saturation of the dynamo at a given field strength results when the production of the field (through induction by the motion of the electrically conducting fluids) balances its Ohmic dissipation, but both of these depend sensi- 
tively on the properties of the flows and fields and for realistic flows it is not generally possible to predict a priori how this balance will be achieved. In many astrophysical contexts, a commonly employed estimate is that the magnetic energy will reach equipartition with the convective kinetic energy (or with whatever flow is responsible for maintaining the field) - see, e.g., Cattaneo (1999), Browning (2008), Featherstone et al. (2009) for example discussions in the context of stellar dynamos, Passot et al. (1995) in the context of turbulence in the interstellar medium, Kulsrud et al. (1997) in relation to galactic magnetism, or Thompson \& Duncan (1993) in regards to magnetars. This is motivated in some cases by the fact that in a closed system with no dissipation, the sum of magnetic and kinetic energies is identically conserved, so any growing field must come at the expense of the flows; in these systems the initial kinetic energy of the flows provides a firm upper bound on the achievable field strengths. But stellar dynamos are not closed systems, and the kinetic energy of the flows may be continually replenished by the vast reservoirs of potential energy present in the system. It is also possible to arrive at the equipartition estimate by considering plausible balances of forces. For example, if the Lorentz force $\mathbf{j} \times \mathbf{B}$ (involving the magnetic field $\mathbf{B}$ and the current density $\mathbf{j}=(c / 4 \pi) \boldsymbol{\nabla} \times \mathbf{B})$ is approximated simply by $j B \sim B^{2} / L$ and balances inertial terms, which are taken to scale as $\rho v \cdot \nabla v \sim \rho v^{2} / L$, we recover $B^{2} \sim \rho v^{2}$ (see, e.g., Roberts 2009). But other seemingly plausible force balances (e.g., of Lorentz and Coriolis forces) can yield estimates of the field that are much greater than the field in equipartition with convection. Indeed, there are many examples of dynamos in which the magnetic energy is believed to be vastly in excess of the convective kinetic energy; the most well-known example is the Earth, where the magnetic energy may exceed the convective energy by factors of 100 or more (e.g., Roberts \& King 2013; Stelzer \& Jackson 2013). Thus, even some of the very strong fields considered in, e.g., MacDonald \& Mullan (2015), which possess energy densities that are far greater than the energy in convective motions (but less than the rotational or thermal energies), even if in our opinion unlikely, are difficult to rule out conclusively on the basis of dynamo theory alone.

Here, we set aside the difficult question of how such fields might be built, and assess instead whether they could survive for extended times and hence have any observable impact. We examine magnetic buoyancy and Ohmic dissipation as complementary constraints on the field distribution in these stars. One of our basic goals is to determine whether fields of the strength envisioned in some models $\left(>10^{6} \mathrm{G}\right)$, however generated, could persist in stellar interiors. More generally, although we are motivated partly by the observation of possible radius inflation in these stars, we aim to explore what limits can be placed on internal field strength without recourse to arguments about equipartition or the specifics of dynamo action in a particular object. We are not the first authors to consider the problem of low-mass star magnetism in this light: some of the possible constraints arising from magnetic buoyancy, for example, were explored by FC14 and Mullan \& MacDonald (2001). In particular, FC14 noted that simple estimates of the buoyant properties of strong fields suggest that simple flux tubes of $>10^{6} \mathrm{G}$ would need to have very small cross-sectional radii to avoid rapid buoyant rise; if they did rise, they would plausibly traverse the stellar interior on timescales of days to weeks. For this and other reasons, FC14 ultimately concluded that such strong field strengths were unlikely. Our work here serves partly to place this analysis on firmer ground, both by examining what scales of field are incompatible with the constraints of dissipative heating, and by comparing the rapid buoyant rise time to the (in some cases also quite rapid) regeneration times that are possible on small scales.

In $\S 2$ we briefly review the magnetic buoyancy of isolated flux tubes, the instability of tubes initially in equilibrium, and the breakup of magnetic layers through buoyancy instabilities. For some very simple field configurations, we give estimates of the time it would take for fields to rise to the stellar surface through the action of such buoyancy instabilities; we also assess under what circumstances a more general field configuration might be stable to these processes. We compare these to orderof-magnitude estimates of the fastest timescales on which the field might plausibly be regenerated to derive limits on the field strength at a given spatial scale. Because smaller-scale fields tend to be less susceptible to buoyant rise, we also calculate for some specific field strengths (e.g., those explored in the MM and FC2014 models) the largest possible spatial scale the magnetism could have in a steady state. In $\S 3$ we briefly verify some of our analytical estimates by turning to MHD simulations of the rise of thin flux-tubes. We confirm the basic results of $\S 2$, specifically that smaller tubes rise slower and that very strong, larger-scale fields rise on remarkably short timescales.

In $\S 4$ we consider complementary constraints arising from the Ohmic dissipation of the fields. We estimate the total power arising from the dissipation of the fields considered in $\S 2$, which again depends on both the strength of the field and its spatial structure. In extreme cases the dissipated energy would greatly exceed the total stellar luminosity, so we conclude that these combinations of strength and morphology are unrealistic. We use this to draw some tentative conclusions about the maximum possible field strengths achievable in stars and sub-stellar objects in $\S 5$, and regard Figure 10 there as the main result of this paper. We briefly discuss some possible extensions of this analysis to other masses in $\S 6$, and close in $\S 7$ with a summary of our work and its main limitations.

\section{MAGNETIC BUOYANCY AS A LIMIT ON FIELD STRENGTHS AND MORPHOLOGIES}

Magnetic fields exert a force, and this force is often decomposed into terms corresponding to a magnetic pressure $\left(B^{2} / 8 \pi\right)$ and to tension along the fieldlines $(\mathbf{B} \cdot \nabla \mathbf{B})$. (The latter is not precisely analogous to tension on a string - see, e.g., Kulsrud (2005) - but the identification of these terms with tension and with pressure is commonplace and we will employ it here.) Because the fields exert a pressure, magnetized gas in total pressure equilibrium with unmagnetized (or less magnetized) surroundings will in general have a different density and/or temperature than those surroundings. These density or temperature differences can in many instances lead to instability, whether the field is composed of discrete struc- 
tures or is instead continuously distributed. The resulting magnetic buoyancy instabilities have been studied for decades both analytically and using numerical simulations. In this section we briefly review the extensive literature on these instabilities, and assess their possible relevance for the fields examined in, e.g., the FC2014 and MM models. We draw extensively on the reviews by Hughes \& Proctor (1988), Hughes (2007), Fan (2009) and Cheung \& Isobe (2014) in what follows. Readers who are already familiar with the buoyancy instability of both isolated flux tubes and smooth layers could proceed to $\S 2.3-2.4$, where we use these concepts to constrain possible field strengths and morphologies in low-mass stars.

Arguably the simplest configuration that exhibits magnetic buoyancy is an isolated, magnetized "flux tube," considered to be in pressure and thermal equilibrium with its field-free surroundings (Parker 1955, 1975). Such structures will have a density deficit relative to their surroundings (as demonstrated in more detail below), and will therefore tend to rise. As the tube rises, it will be buffeted by convective flows that can help or hinder its progress; in general, whether the tube can rise coherently, and if so at what rate, is a function of both the surrounding convective velocity field and the properties of the flux tube itself. In many simple models, the terminal velocity attained by the tube is related to the Alfven velocity $\left(v_{a}=B / \sqrt{4 \pi \rho}\right)$, multiplied by factors that depend on the size of the flux tube and the background stratification.

How such flux tubes might arise is still a matter of some uncertainty. Some authors have argued that the natural state of the dynamo is a collection of fibril magnetic tubes - e.g., Parker (1984) argued that this configuration is a minimum-energy state for a given field strength. Others have shown (as demonstrated below) that even smooth layers of magnetic field may break up and yield more compact structures that resemble flux tubes. Still other simulations have self-consistently generated magnetic structures from the combined action of convection and shear (e.g., Nelson et al. 2011, 2014) that rise due to buoyancy and advection by surrounding flows, in a manner somewhat similar to simple flux tubes. In any case, it has proven useful in many contexts to study the properties of such idealized tubes as a proxy for more complex field configurations.

Parker (1975) pointed out that the rapid rise of such tubes poses major problems for simple models of the solar dynamo. The rise time of an isolated tube is estimated to be much shorter than the length of the solar cycle, and shorter than the timescale on which the field could plausibly be regenerated by stretching amidst the convection zone. This led Parker to argue that the global solar dynamo might be situated at the base of the convection zone (where rise times are larger), and to point out that fields could be amplified to much greater strengths (before becoming susceptible to buoyancy instabilities) in the stably stratified region below. This, coupled with the later discovery using helioseismology of a "tachocline" of shear at the interface between the convective envelope and radiative interior (e.g., Thompson et al. 1996), helped lead to the now-prevalent "interface dynamo" paradigm for global field generation in the Sun (see, e.g., Parker 1993; Ossendrijver 2003; Miesch 2005). This picture has been critically reex- amined by some in recent years, with various numerical simulations suggesting that fairly strong large-scale magnetic fields could be generated within the convection zone while evidently avoiding severe losses by magnetic buoyancy (see, e.g., discussions in Brun et al. 2013; Brown et al. 2011; Brandenburg 2005). But the basic reality of magnetic buoyancy is undisputed, even though downward "magnetic pumping" (Tobias et al. 2001) and other effects can limit its effectiveness in some circumstances.

Below, we essentially adapt the argument of Parker (1975) for the case of very strong fields in fully convective stars, incorporating what is now known about the rise of simple flux tubes and the instability of smoother field distributions. We begin by calculating the rise time of the simple, thermally isolated flux tube described above, then summarize results from more complex calculations that incorporate the effects of radiative transfer, interaction with the surrounding turbulence, and other effects. We describe the conditions under which smooth field distributions are unstable to similar buoyancy instabilities, and apply this to conclude that some previously studied profiles for the interior magnetism in low-mass stars are unstable. We then estimate plausible regeneration times for the magnetism under a variety of assumptions, and use this to assess the strength and size of fields that could likely be regenerated faster than they are lost from magnetic buoyancy.

\subsection{Simple estimates of rise times}

We first consider the rise of an unbent, untwisted, isolated flux tube in thermal equilibrium with its surroundings. It is worth noting that this is not in itself an instability in the usual sense (there is no initially stable configuration that is being perturbed); nonetheless this simple configuration has many features in common with the more complex scenarios described below. In this case, for the flux tube to remain in pressure equilibrium with its surroundings, we must have

$$
P(z)=P_{i}(z)+B^{2} / 8 \pi
$$

with $P_{i}$ the pressure internal to the tube and $P$ the external pressure. If the temperatures inside and outside the tube are the same, then the density within the tube must be smaller than that of the surrounding medium,

$$
\rho(z)-\rho_{i}(z)=\rho(z) B^{2}(z) / 8 \pi P(z),
$$

and this reduced density results in a buoyant force per unit length $F \sim \pi a^{2} g\left(\rho-\rho_{i}\right)$, with $a$ the cross-sectional radius of the tube. Parker (1975) assumed the upward acceleration of the tube is resisted primarily by the aerodynamic drag, $F_{d}=\frac{1}{2} \rho u^{2} a C_{D}$, with $C_{D} \sim 1$ the drag coefficient. With this assumption, the terminal velocity occurs for $F=F_{D}$, and hence the rise velocity is

$$
u=V_{a}\left(\frac{\pi a}{C_{D} H_{p}}\right)^{1 / 2}
$$

where $V_{a}=B / \sqrt{4 \pi \rho}$ is the Alfven speed and $H_{p}=$ $P /(g \rho)$ is the local pressure scale height. Later authors have adopted many other prescriptions, considering for example the turbulent diffusivity of the medium as the primary impediment to the tube's rise, but for the very 


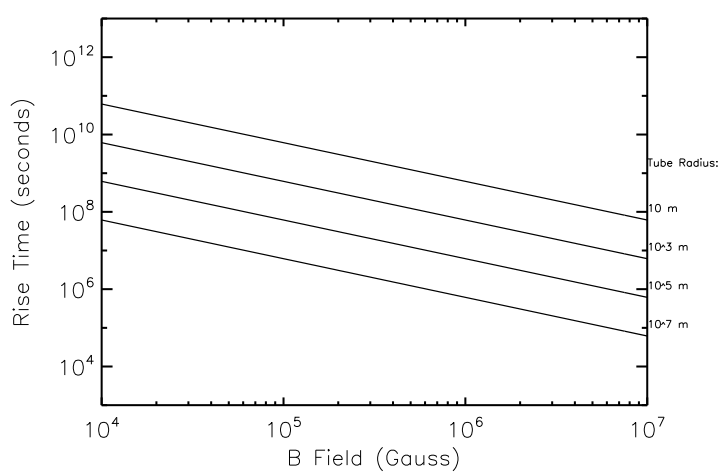

Figure 1. Simple estimate of rise time (eqn 3) for isolated flux tubes of indicated sizes and strengths. Stronger, larger-scale tubes rise more quickly than weak, small-scale fields.

strong fields considered here these changes have only minor effects on the total rise time.

In Figure 1, we use this simple estimate of the rate of rise of isolated flux tubes to assess the rise time $t_{\text {rise }}=(R-r) / u$ for tubes of various sizes and strengths. We have adopted stratifications from a 1-D model of a 0.3 solar-mass M-dwarf, provided for us by Isabelle Baraffe and computed following Chabrier \& Baraffe (1997). For simplicity and illustrative purposes we will in some discussions take $H_{p}$ to have a constant, representative value equal to its value at $r=0.25 R\left(H_{p} \approx 8 \times 10^{9} \mathrm{~cm}\right)$, but the calculations shown in Figure 1 include the spatial variation of all quantities, integrated numerically to calculate the rise time for tubes starting at the origin. The rise time decreases with increasing field strength, and is longer for small-scale fields than for large-scale ones. This is essentially because the rise time in this simple model is set by a balance between drag forces (which increase with the surface area of the tube) and buoyancy forces (which increase with its volume), so thick tubes rise faster than thin ones in accord with their larger ratio of volume to surface area. In what follows, it is useful to note that the rise time for deep-seated fields in this model is given by

$$
t_{\text {rise }} \sim \frac{R}{u}=\frac{R}{C a^{1 / 2} B}
$$

where $C=1 / \sqrt{4 \rho H_{p} C_{D}}$ varies slowly with radius but is typically about $6 \times 10^{-7}$ in cgs units.

This simple calculation neglected heat transfer between the rising tube and the surrounding medium, among other effects. Many other authors (e.g., Schuessler 1977; Spruit 1981b; Spruit \& van Ballegooijen 1982; Moreno-Insertis 1983, 1986; Fan et al. 1993; Caligari et al. 1995, 1998) have carried out more sophisticated calculations that examine the behaviour of such tubes under different assumptions. If, for example, the tube does not equilibrate instantly to the surrounding temperature as it rises, but instead expands adiabatically along its ascent, then its rate of rise depends on the properties of the external medium. If the medium is unstably stratified (i.e., convective), then the tube still must rise indefinitely; if instead the surroundings are isothermal, for instance, it is possible for the flux tube to reach the same density as its environment and hence attain mechanical equilibrium. In practice, tubes are likely neither to equilibrate instantly to their surrounding temperature nor to behave perfectly adiabatically; the reality will be somewhere between these two extremes, determined by the rate at which heat flows in or out of the tube. It is possible to show that the rise time is fastest for tubes that rise adiabatically, and slowest for those that adjust instantly to the surrounding temperature (see, e.g., Moreno-Insertis 1983, Fig 1). The rise times displayed in Figure 1 are thus arguably somewhat conservative estimates of the rate at which isolated tubes might rise.

Some additional insight can be gained by considering the case of a thin magnetic tube initially in thermal or mechanical equilibrium with its surroundings, and asking under what circumstances this equilibrium is stable. For mechanical equilibrium to be possible in the first place, initially the tube must (for the reasons given above) be at a different temperature than its surroundings, so in this case stronger fields have the slightly bizarre effect of forcing the initial tube to be cooler. In this special case, it would be possible to maintain very strong fields even amidst an unstably stratified region if the tube were required to remain straight (see Spruit \& van Ballegooijen 1982; Moreno-Insertis 1983). But if the field lines are allowed to bend, then (as noted by Parker 1955, analyzed by Spruit \& van Ballegooijen 1982 analytically, and confirmed numerically in many later papers) a flow away from the crests of the tube tends to enhance the buoyancy of those regions, leading again to instability on sufficiently long wavelengths.

As a flux tube rises, it will be affected by the convective flows in a variety of ways. Arguably the simplest approach, as adopted above, is to assume that the flux tube's motion is resisted by aerodynamic drag. Other authors have modeled the braking effect of the surrounding turbulence in other ways, for example as an eddy viscosity (e.g., Unno \& Ribes 1976; Schuessler 1977; Moreno-Insertis 1983), leading in some cases to significantly longer rise times. The drag in the turbulent viscosity models tends to be greater than that in models assuming aerodynamic drag if the velocity of the rising flux tube is small compared to the convective velocity (e.g., Moreno-Insertis 1983); for fields (like those considered here) that are much stronger than the convective flows, turbulent viscosity cannot greatly impede the flux tube's rise. Later work using numerical simulations has extensively investigated the rise of both tubes and more general magnetic structures along with (in some cases) their interaction with convective flows, whether imposed, self-consistently generated, or simply parametrized. Examples are provided by Hughes \& Falle (1998); Wissink et al. (2000); Cattaneo et al. (2006); Cheung et al. (2006); Jouve \& Brun (2009); Favier et al. (2012); Barker et al. (2012); Pinto \& Brun (2013); Martinez-Sykora et al. (2015). The processes by which magnetic fields are more generally expelled from regions of active convection has also been extensively studied; see Tobias et al. (1998, 2001, 2008); Weiss et al. (2004) for examples. In general, for flux tubes to survive passage through the convection zone in these models they must exceed the value in equipartition with the convection by a geometrical factor of order $H_{\rho} / a$, where $a$ is the cross-sectional radius of the flux tube: in other words, at fixed field strength, small flux tubes are more easily held down by the convection. 
The qualitative trends revealed by comparatively simple estimates - that strong, thick tubes rise faster than weak or thin ones - largely appear to be realized for somewhat more complex flux tube configurations as well, even when a panoply of effects not present in Parker's original formulation are included. Further, while the thin flux tube approximation itself suffers from significant limitations (see, e.g., discussion in Hughes 2007), its estimates appear to capture the overall rate of rise of buoyant fields reasonably well, at least when compared to full MHD simulations of rising field structures in certain regimes (Cheung et al. 2006). We therefore adopt these estimates in much of our discussion here, while recognizing that variations in how the tube exchanges heat with its surroundings, interacts with the convection, and expands with height, are all likely to affect our conclusions quantitatively at some level. In $\S 3$, we will turn to our own thin flux-tube MHD simulations, which include some of these effects (albeit still in a very simplified way) to assess how robust our estimates may be.

\subsection{Instability of magnetic layers}

Just as isolated flux tubes represent one limit of possible field configurations, another widely studied limit involves a global distribution of magnetism, varying initially with depth in some specified way. Here we briefly review the extensive literature on buoyancy instabilities of both discontinuous layers of field (i.e., "slabs" of magnetism) and continuous field distributions.

In general, an atmosphere that contains a layer of magnetic field underlying an unmagnetized (or lessmagnetized) fluid is unstable to buoyancy instabilities. In essence, the presence of the magnetic field makes the fluid "top-heavy," because heavier (less-magnetic) gas is being supported by lighter (more magnetic) gas, so these instabilities often take on the same character as classical Rayleigh-Taylor instability.

It is useful to distinguish between two broad types of disturbances, known as "undular" and "interchange" modes. Interchange modes are those that involve no bending of field field lines (i.e., $k$ is perpendicular to $B$ ), while purely undular modes have $k$ parallel to $B$. In general, perturbations may be fully $3-\mathrm{D}$ and have elements of both undular and interchange modes. The growth rates of the interchange modes increase uniformly with increasing wavenumber, limited ultimately only by dissipation. The undular modes, on the other hand, have maximum growth rate at finite and relatively small wavenumbers (i.e., large spatial scales), because small scales are stabilized by magnetic tension. Because of this, the interchange modes generally have much faster linear growth rates than the undular modes (e.g., Nozawa 2005; Cheung \& Isobe 2014). The nonlinear stage of the instability, however, may be dominated by the undular (or mixed) modes in many circumstances. The linear growth rates for these types of instabilities are of order $H_{p} / v_{a}$ in the absence of diffusion and rotation (Acheson 1979), implying that for the field strengths under consideration here, they would develop on timescales that are short relative to both the large-scale convective overturning time and to the rise time of small flux tubes.

For the interchange modes, it is straightforward to show that a necessary and sufficient condition for insta- bility is

$$
\frac{v_{a}^{2}}{\gamma H_{P}} \frac{d}{d z} \ln \left(\frac{B}{\rho}\right)<N^{2}
$$

where $N$ is the Brunt-Vaisala (buoyancy) frequency and other symbols take their usual meanings (Acheson 1979; Hughes \& Proctor 1988; Newcomb 1961). Thus, even in a convectively stable atmosphere, instability will occur if $B / \rho$ decreases rapidly enough with height; in a neutrally or unstably stratified layer, any decrease in $B / \rho$ with height is unstable.

The window of instability for undular modes is even broader. These modes (in which the field lines are bent rather than remaining straight) can occur in circumstances where the interchange modes are still stable, essentially because in the undular case the instability can (by compressive motions along the field lines) avoid doing extra work against magnetic pressure while tapping into the potential energy of the stratification (Hughes \& Cattaneo 1987). These modes are thus the smooth-field analogue to the "bent flux tubes" discussed above, and (as in that case) are unstable in some regimes where the interchange modes are not. As shown by Newcomb (1961) using an energy priciple, and analyzed by Parker (1966) using normal modes (see also Thomas \& Nye 1975), instability occurs (in the absence of dissipation) if

$$
\frac{1}{\gamma H_{P}} \frac{d}{d z} \ln B<\frac{k_{y}^{2} k^{2}}{k_{x}^{2}}+\frac{N^{2}}{V_{a}^{2}}
$$

where $k_{x}$ and $k_{y}$ are horizontal wavenumbers (with $k_{y}$ the wavenumber along the tube; recall that for the interchange modes $k_{y}=0$ ), and $k$ is the total wavenumber. Thus while the interchange modes require $B / \rho$ to decrease with height (or increase with depth), the undular modes require only that $B$ decrease with height. On the other hand, they are also stabilized at short wavelengths by magnetic tension; for field distributions that vary slowly with height, only global-scale perturbations are likely to be unstable.

These instabilities have, like their isolated flux tube equivalents, been studied extensively using numerical simulations. The linear growth and subsequent evolution of pure interchange modes (sometimes called the "magnetic Rayleigh-Taylor" instability) were examined by Cattaneo \& Hughes (1988), for example; the behavior of the pure undular mode (Parker instability) was studied by Shibata et al. (1989a, b) and Fan (2001), among others. Many subsequent papers have examined the general nonlinear behavior of layers of field subject to arbitrary (mixed-mode) perturbations (e.g., Matsumoto \& Shibata 1992; Matthews et al. 1995; Wissink et al. 2000; Kersalé et al. 2007). In general, the effect of these instabilities is to cause an initially uniform layer to break up into smaller features that then evolve nonlinearly under the competing influences of buoyancy, magnetic tension, and other effects. Thus for example in the solar dynamo, it is widely thought that these processes may play a role in generating magnetic structures akin to "flux tubes" from an initially smooth field in the tachocline (e.g., Cheung \& Isobe 2014).

It is helpful to consider the magnetic field profiles studied in FC2014 in light of these instability criteria. For 


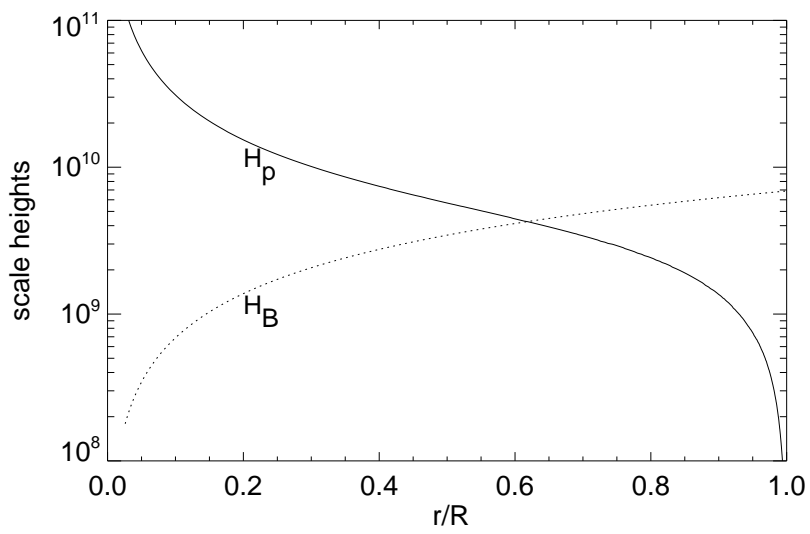

Figure 2. Analysis of the instability of previously proposed smooth magnetic field profiles via undular and interchange modes. Shown is a comparison of $H_{\rho}=-\rho /(d \rho / d r)$ in a 1-D stellar model and $H_{B}=-B /(d B / d r)$ for part of the FC2014 "dipole" field profile. (The FC2014 profile departs from this curve for $r / R<0.15$, where $B$ increases with $r$.) The regions where $H_{B}<H_{\rho}$ (i.e., interior to $r \approx 0.6 R$ ) are potentially unstable to both undular and interchange modes. All regions exterior to $r / R=0.15$ (i.e., the radius at which the field strength begins to decline with radius) are unstable to undular modes at some wavelengths.

specificity, consider their "dipole" field profile, which reaches a specified maximum amplitude (of order $10^{7} \mathrm{G}$ in some cases) at a radius $r_{t}=0.15 R$, and falls off like $1 / r^{3}$ away from that point. By inspection, this profile is unstable to undular modes (which require only that $B$ decrease with height) at all radii exterior to $r_{t}$, but these modes could in principle be suppressed at short wavelengths. In Figure 2, we further compare the gradients of the magnetic field and of density, $d(\ln B) / d r=$ $B^{-1}(d B / d r)$ and $d(\ln \rho) / d r$; recall that if the field declines more rapidly than the density (i.e., $d(\ln B) / d r$ is greater in magnitude than $d(\ln \rho) / d r)$, the profile is unstable to pure interchange modes as well. For convenience, we have expressed these as scale heights, plotting $H_{B}=-B /(d B / d r)$ and $H_{\rho}=-\rho /(d \rho / d r)$; instability to interchange modes thus requires $H_{B}<H_{\rho}$. In the region exterior to $r_{t}$ (i.e., where the field is falling off with height), $H_{B}=r / 3$, whereas density falls off less rapidly out to a fractional radius $r \approx 0.6 R$. Thus the profile is unstable to interchange modes (at all wavelengths) over a substantial fraction of the interior. The same would be true of the "Gaussian" profiles also employed in FC2014, for which $B$ declines more steeply than $\rho$ throughout virtually the entire domain exterior to $r_{t}$. We therefore conclude that a smooth field distribution whose radial variation is given by either of these profiles would be unstable to both interchange and undular modes of magnetic buoyancy instability over a large fraction of the interior, and would therefore break up into smaller-scale magnetic structures. Although the nonlinear evolution of these structures is likely to be complex, prior 3-D simulations of these instabilities have suggested that the ensuing behavior resembles that of simple flux tubes in some important respects, namely that the strongest-field regions tend to rise buoyantly unless they are "pumped" downwards by convection.

Because the specific radial profiles employed in FC2014 are somewhat arbitrary, it is also instructive to examine the possible instability of more general field distribu- tions. Any profile $B(r)$ that increases with depth will be unstable to undular modes, but the linear growth rates of these modes can be relatively slow (since they occur only on large spatial scales), and we find it difficult to predict with certainty whether such instability would act ultimately as a loss mechanism for magnetic energy in the presence of many other competing effects. Consider, then, the instability to interchange modes, which occur on all scales and hence grow more rapidly. If we suppose that $B(r)$ increases smoothly from an observed surface value $B_{\text {surf }}$ to some interior maximum $B_{\max }$, instability to interchange modes is inevitable if $B_{\max } / B_{\text {surf }}$ exceeds a value of order $\rho_{\max } / \rho_{\text {surf }}$. The radial surface on which we can constrain $B_{\text {surf }}$ is not just the stellar photosphere (where $\rho$ is very small), but somewhat deeper. In the Sun, for example, although the radial extent of large active regions is still a matter of considerable debate, sunspots are coherent enough to support magneto-acoustic modes of oscillation down to a depth of at least $10 \mathrm{Mm}$ (e.g., Kosovichev 2002; Kosovichev \& Duvall 2006); this in effect constrains the field in the outer $10 \mathrm{Mm}$ of the solar convection zone to be no stronger than the fields observed in spots. In convective M-dwarfs, observations suggest surface fields of no more than a few kG (e.g., Johns-Krull \& Valenti 1996; Donati et al. 2006), and it is reasonable to assume that no stronger fields than this exist in the outer few $\mathrm{Mm}$ of the star. For specificity, if the field at $0.95 R$ (i.e., less than $10 \mathrm{Mm}$ below the surface) is constrained to be no more than $5 \mathrm{kG}$ by observations, then $B(r) / \rho(r)$ cannot exceed its value at that radius without triggering instability to interchange modes. In a sample 1-D model of a 0.3 solar-mass main-sequence M-dwarf provided to us by Isabelle Baraffe (Chabrier \& Baraffe 1997), the density at $0.95 R$ is about $0.9 \mathrm{~g} \mathrm{~cm}^{-3}$, whereas in the interior it reaches a maximum of about 100 times this $\left(\rho_{\max }=92 \mathrm{~g}\right.$ $\mathrm{cm}^{-3}$ ), so to avoid instability $B$ must not exceed values of order $100 \times B_{\text {surf }} \approx 5 \times 10^{5} \mathrm{G}$ anywhere in the interior. If this limit is violated, it is difficult to avoid the conclusion that even smooth layers of field would quickly break up from buoyancy instability, with a subsequent nonlinear evolution that includes buoyant rise towards the surface.

\subsection{Regeneration times for the magnetism}

The buoyant rise of strong magnetic fields represents a mechanism by which these fields may be "lost" from the deep interior. But we also expect that convection, rotation, and shear within the star will together act to regenerate the field through dynamo action. In this section, we briefly examine the timescales on which this regeneration might occur.

A simple estimate of the characteristic timescale for field growth can be derived by examination of the induction equation, which in the absence of dissipation is

$$
\frac{\partial \mathbf{B}}{\partial t}=\nabla \times(\mathbf{v} \times \mathbf{B})=-\mathbf{v} \cdot \nabla \mathbf{B}+\mathbf{B} \cdot \nabla \mathbf{v}
$$

where all symbols take their usual meanings. Qualitatively, the first term on the right hand side represents advection of the magnetism by the flow; the second term captures stretching of fieldlines by a non-uniform flow.

If we first suppose that the field and flow can each 
be characterized by just one spatial scale $(L)$ and one timescale $t_{\text {gen }}$, then the induction equation suggests

$$
\frac{B}{t_{\text {gen }}} \sim \frac{V B}{L} \rightarrow t_{\text {gen }} \sim \frac{L}{V} .
$$

Thus in the absence of dissipation and any nonlinear feedback on the flow, we expect that the field will grow on a timescale of order the convective overturning time. Much more sophisticated limits can be derived by turning to the theory of fast dynamos - i.e., dynamos whose growth rate is non-zero in the limit of zero diffusion (see, e.g. Childress \& Gilbert 1995, for review). It was conjectured by Finn \& Ott (1988), and later proven by Klapper \& Young (1995) in certain circumstances, that the growth rate for kinematic dynamos is bounded above by the topological entropy of the flow (which is in turn a bound on the Lyapunov exponents of the flow, and physically is related to the rate at which trajectories in a chaotic flow diverge). In practice, applying this limit is not particularly useful for our purposes: the problem we consider here is neither purely kinematic nor free of diffusion, and moreover there is no obvious way to estimate the topological entropy of an arbitrary convective flow. Fortunately, numerical simulations of dynamo action in a wide variety of contexts suggest that the simpler estimate above $\left(t_{\mathrm{gen}} \sim t_{\mathrm{dyn}}\right.$, suitably defined) is a reasonable upper bound on the rate at which magnetic energy grows in the kinematic phase (e.g., Cattaneo \& Hughes 2006). Field growth in the nonlinearly saturated phase is likely to be considerably slower.

We will adopt this simple bound in our discussion below, but a few additional points require specification. These relate to what is assumed about the flow, and about the lengthscales on which it and the field are assumed to vary.

We will assume, first, that the growth of fields is limited by appropriate convective timescales, rather than by timescales associated (say) with some unknown internal shear flow. This is not because shear is guaranteed to be dynamically unimportant; indeed, as we note below, it is conceivable that internal differential rotation might play as significant a role as the convection. But no purely toroidal flow (such as that represented by differential rotation) can act as a dynamo on its own (see discussions in Bullard \& Gellman 1954, Jones 2008). Another flow must act to generate poloidal field from toroidal, and in general the overall rate of growth will be limited by the slower of these. The most plausible candidate for the latter step is the convection, so we assume it is the stretching properties of this flow that will limit how quickly a general field can be rebuilt.

Convection in stellar interiors occurs over a wide range of spatial and temporal scales, so estimates of the convective overturning time (and hence the field regeneration time) are fraught with some uncertainty. The simplest model is that the regeneration process must ultimately occur on the turnover time of the comparatively largescale, slowly overturning eddies in the convection, since these carry most of the energy. Mixing length estimates (e.g. Hansen \& Kawaler 1994; Thompson \& Duncan 1993) and simulations of stellar/planetary convection (e.g., Abbett et al. 1997; Browning et al. 2004; Meakin \& Arnett 2007; Viallet et al. 2013) both suggest that these eddies should have velocities that scale roughly as $v_{c} \sim(F / \rho)^{1 / 3}$, with $F$ the energy flux that must be carried by convection, and should have typical sizes of order a pressure scale height (which is in turn of order the stellar radius $R$ ) in the deep interiors of these stars. Written explicitly, we have

$$
t_{\text {gen }}^{\text {slow }} \sim \frac{d}{v_{c}} \sim \frac{d}{(F / \rho)^{1 / 3}},
$$

defining the depth $d=R-r$.

This estimate (which we refer to below as the "slow" model) involves velocities in the interior of a 0.3 solar mass star that are of order a few $\mathrm{m} \mathrm{s}^{-1}$, and hence implies overturning times of order $10^{8}$ seconds, or about 3 years. Turning again to Figure 1, we see that if this is the timescale on which fields are regenerated, very strong flux tubes (of more than say $10^{6} \mathrm{G}$ strength) have rise times shorter than the regeneration time unless they have very small radii $\left(a \leq 10^{3} \mathrm{~m}\right)$.

But the convection likely contains eddies with a wide range of spatial scales, and the smallest of these eddies plausibly overturn much more quickly than the largescale motions modeled in MLT. Although these smallerscale eddies do not contain much energy, it is worth considering the timescales associated with field regeneration on these smaller scales. This is also the timescale on which a large-scale flow acting on a pre-existing smallscale field could produce fluctuations on that same small scale. We consider a simple model where a large-scale velocity field $v_{c}$ is taken to act on fields at all scales $a$, with a characteristic regeneration time

$$
t_{\text {gen }}^{\text {fast }} \sim \frac{a}{v_{c}} \sim \frac{a}{(F / \rho)^{1 / 3}} .
$$

We retain this estimate mainly as an upper limit to how quickly fields could be regenerated for a short time by large-scale flows (e.g., shear, discussed more below). We refer to this estimate as the "fast eddy/shear" model.

It is also possible to define a scale-by-scale overturning time taking into account the variation of the flow with scale; that is, assuming that fields at each scale are built by motions at the same scale. The scale dependence of the velocity in rotating, magnetized convection is still a matter of considerable debate (e.g., Kraichnan 1965; Grappin et al. 1982; Sridhar \& Goldreich 1994; Goldreich \& Sridhar 1995; Biskamp \& Müller 2000; Boldyrev 2006; Beresnyak \& Lazarian 2010; Perez et al. 2014), and in all cases these small-scale eddies presumably possess less kinetic energy than somewhat largerscale flows (since the velocity $v^{l}$ on scale $l$ decreases with decreasing $l$ ), so our estimates here are highly uncertain. We consider a scale-dependent velocity field $v^{l}$ that scales with $l$ as

$$
v^{l}=v^{0}\left(\frac{l}{l^{0}}\right)^{\alpha}
$$

where $v^{0}, l^{0}$ refer to the velocities on some large length scale $l^{0}$. The power-law index $\alpha$ would be $1 / 3$ for turbulence obeying a Kolmogorov-like cascade. In this model the turnover time, which we will take as representative of the regeneration time for fields on scale $a$, scales like

$$
t_{\text {gen }} \sim \frac{a}{v_{c}^{a}} \sim \frac{a}{(F / \rho)^{1 / 3}(a / R)^{\alpha}},
$$


where we have further assumed that the large-scale eddies (whose scale-dependent amplitude $v_{c}^{a}$ is given by mixing-length theory) have a spatial scale of order the stellar radius $R$ (which is of order the pressure scale height in the deep interior). We refer to this as the "cascade" model below.

\subsection{Resulting constraints on field strengths and morphologies}

Our analysis thus far has suggested that fields of the strength envisioned in some previous models (MM01, FC14) are likely to be unstable. If the magnetism consists of smooth layers, it is apt to break up into smaller-scale structures unless the stability criteria outlined above are satisfied; these instabilities are generally very rapid (e.g., Acheson 1979). If the field consists of bundles of discrete flux tubes it is again unstable, on a timescale that varies with the geometry and strength of the field.

In both cases, the instability acts as a loss mechanism for fields in the deep interior, acting over some characteristic time $\tau_{B}$. If the field is not regenerated on a comparable timescale, then the overall level of magnetic energy must decline. For the proposed magnetic field profiles to represent steady-state solutions at the present day, the star would need to produce field in the interior at least as quickly as it is carried away by buoyancy instabilities.

Denoting the buoyant rise time for fields of given strength $B$ and spatial scale $a$ by $t_{\text {rise, and the regen- }}$ eration time for the same fields by $t_{\text {gen }}$, we can therefore equate $t_{\text {rise }} \sim t_{\text {gen }}$ to find a crude estimate of the maximum field that could be sustained indefinitely. In Figure 3 , we show this maximum field strength as a function of radius for simple flux tubes (i.e., tubes obeying eqn 3) of various sizes, assuming that the regeneration timescale is given by the overturning time of the slow, large-scale convective eddies (i.e., the "slow eddy" model $t_{\text {gen }} \sim d / v_{c}$, with $v_{c}$ varying with depth as described above). In this case

$$
\frac{t_{\text {gen }}}{t_{\text {rise }}} \sim \frac{d / v_{c}}{d / C a^{1 / 2} B}=\frac{C a^{1 / 2} B}{v_{c}} \sim 1
$$

where $C$ is as described earlier, so in equilibrium the field in tubes of size $a$ is given by

$$
B \sim \frac{v_{c}(r)}{C(r) a^{1 / 2}} .
$$

This is, to order of magnitude, the same as the minimum field needed for fields at a given scale to overpower the effects of downward magnetic pumping by the convection. For example, Fan (2009) estimates the latter as

$$
B \geq \frac{2 C_{D}}{\pi}\left(\frac{H_{p}}{a}\right)^{1 / 2} B_{\text {eq }}
$$

where $B_{\text {eq }} \approx \sqrt{4 \pi \rho} v_{c}$ is the field strength in equipartition with large-scale convective eddies. In essence, this just says that fields that are buoyant enough to overcome downward pumping by convection will always rise faster than they can be regenerated by the large-scale eddies. In this limit, to maintain fields of order $10^{7} \mathrm{G}$ would require that the field be composed of tubes with scale $a \leq 100 \mathrm{~m}$; conversely, for fields of scale $a=10^{7} \mathrm{~m}$,

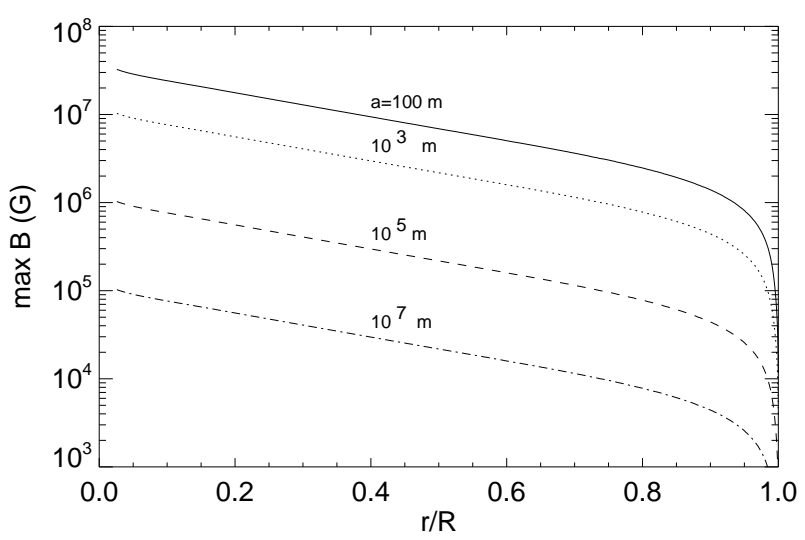

Figure 3. Maximum field strength achievable if large-scale eddies with turnover time $\tau \sim d / v_{c}$ must regenerate field lost due to magnetic buoyancy during the same time. Limits are shown as function of radius for varying flux tube sizes $a$, using the simplest rise time estimate (eqn 3). The maximum sustainable field is larger if the field scale $a$ is small, because the rise time for those fields is long.

only fields with strengths less than about $10^{5} \mathrm{G}$ could be maintained. These estimates vary slightly depending on what is assumed about the radial variation of $H_{p}$ (and with it the quantity $C$ ) and the turnover time; if we ignore the radial variation of $H_{p}$, and adopt a constant value representative of the deep interior instead, the maximum allowable field is reduced by a factor of about three.

If instead we make the generous assumption that scaleby-scale regeneration of the field occurs on a timescale $t_{\text {gen }} \sim a / v_{c}$, taking $v_{c}$ to be a constant (what we called the "fast eddy/shear" model above), then much stronger fields can be maintained on small scales. In this case

$$
B \leq \frac{v_{c} d}{C a^{3 / 2}}
$$

so the maximum field is faster than in the "slow" estimate by a factor of $d / a$, which is large for small $a$. In practical terms this means that fields with strength $10^{7}$ $\mathrm{G}$ are too buoyant unless $a \leq 10^{6} \mathrm{~m}$.

The maximum field strength in the "cascade" model, which has velocities $v_{c}^{a}(r)$ that fall off with decreasing spatial scale according to a power law, is intermediate between these two limits at given $a$ for reasonable choices of the power-law dependence. Specifically, the choice $\alpha=1$ (which gives a constant scale-dependent overturning time $\left.\sim a / v^{a}\right)$ is akin to the "slow eddy" model, whereas the choice $\alpha=0$ (implying the local overturning time decreases linearly with $a$ ) corresponds to the "fast eddy/shear" case. A Kolmogorov-like cascade corresponds to $\alpha=1 / 3$, and the Iroshnikov-Kraichnan spectrum would correspond to $\alpha=1 / 4$, in both cases yielding field estimates that fall between the "slow" and "fast" cases shown in these figures. Written explicitly, the maximum field is of order

$$
B \leq \frac{v_{c}^{R}}{C a^{1 / 2}}\left(\frac{d}{a}\right)\left(\frac{a}{R}\right)^{\alpha},
$$

i.e., it differs from the "slow eddy" estimate by the factors $(d / a)(a / R)^{\alpha}$. (The "slow eddy" expression quoted earlier is slightly different from that derived by taking $\alpha=1$ 
here, simply because we have assumed the relevant scale in the turbulent cascade is $(a / R)$ rather than $(a / d)$.)

Equivalently, we can also use these estimates to construct limits on the maximum characteristic spatial scale of the field, if it is to be stable against buoyancy or regenerated faster than it buoyantly rises. In general, for the "cascade" model of field regeneration with a scale-dependent overturning time $t \sim l / v_{c}(l)$ and taking $v_{c}(l) \propto l^{\alpha}$, we have

$$
a_{\max }=\left[\frac{d}{R^{\alpha}} \frac{v_{c}^{R}}{C B}\right]^{\frac{2}{3-2 \alpha}}
$$

where as before $C=1 / \sqrt{4 \rho H_{p} C_{D}}$ and we are denoting $v_{c}^{R}(r)$ as the velocity on spatial scale $R$ at location $r$. It is perhaps more instructive to rewrite this assuming that $H_{p} \approx R$ (true to order of magnitude in the deep interior of a low-mass star), $C_{D} \approx 1, d \approx R$, and denoting $B_{\text {eq }} \sim$ $\sqrt{4 \rho} v_{c}(R)$ as the magnetic field strength in equipartition with the largest-scale convection, so that

$$
a_{\max } \sim R\left(\frac{B_{\mathrm{eq}}}{B}\right)^{\frac{2}{3-2 \alpha}} .
$$

Written in this way, it is clear that fields of order the equipartition strength are stable against buoyancy, or rise more slowly than they are regenerated, on all spatial scales. Stronger fields (relative to equipartition) are more subject to rapid buoyant losses, but these can be avoided if the characteristic size scale of the field is small enough. How small is small enough depends on the assumed characteristics of the regeneration process. Here $\alpha=0$ corresponds to the extreme "fast eddy/shear" model in which the scale-by-scale turnover time decreases linearly with decreasing spatial scale; in this case $a_{\max } \propto\left(B_{\mathrm{eq}} / B\right)^{2 / 3}$. If regeneration on small scales is somewhat slower, as for a Kolmogorov-like cascade $(\alpha \sim 1 / 3)$ or the "slow eddy" models considered above $(\alpha=1)$, then $a_{\max } \propto\left(B_{\text {eq }} / B\right)^{6 / 7}$ or $\left(B_{\text {eq }} / B\right)^{2}$ respectively.

These limits are assessed in Figure 4, which shows the maximum size $a$ for which thin flux tubes have $t_{\text {rise }}$ longer than $t_{\text {gen }}$. These limits vary somewhat with radius, so we have chosen two representative spots in the interior (at $r=0.15 R$, where the FC2014 "dipole" field reaches its maximum, and $r=0.5 R$ ) for some estimates. The solid and dashed lines give the limits when the relevant overturning time is the large-scale one (i.e., the "slow/eddy" model), and so also correspond to an approximate limit on fields that are "pumped" downward (i.e., fields above the solid line are too large in scale to be pumped downward at any given field strength). This corresponds to the line

$$
a_{\max }=\left(\frac{v_{c}}{B} \frac{1}{C}\right)^{2},
$$

where $C$ is as before. The dotted line (labeled "fast/shear") corresponds to the more optimistic (and in our opinion unrealistic) estimate in which the field is taken to be regenerated on the rapid, kinematic smallscale overturning time $a / v_{c}$. Here the allowable field can be considerably larger in scale in most cases. (An exception occurs at very large $a>d$, because in that case the

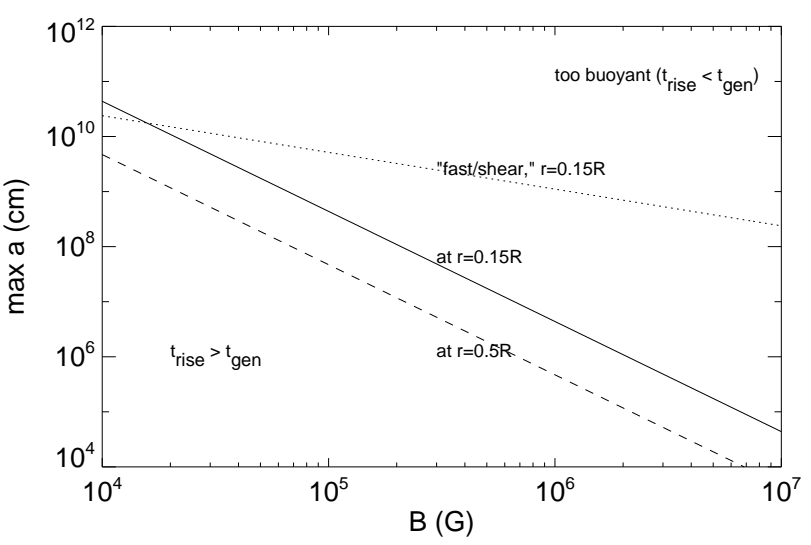

Figure 4. Maximum size of flux tubes at any given field strength for which the rise time is longer than the generation time, for the "fast/shear" and "slow" models considered above. Only very smallscale fields are permissible at high field strengths, because largescale fields rise more rapidly than they can plausibly be regenerated. The solid and dashed lines also correspond to the largestscale fields that could be pumped downward by eddies at a given field strength at that depth (see text). The limits vary somewhat with radius; limits at two representative depths are illustrated for the "slow" regeneration model.

"slow eddy" model actually gives slightly faster regeneration times, $t_{\text {gen }}=d / v_{c}$, than the "fast" model with $t_{\text {gen }}=a / v_{c}$.)

We will adopt the more stringent estimate of equation 20. which we think best reflects limits on the field growth in this regime - and also, importantly, represents limits on a field that is not actively maintained by dynamo action, but is instead passively responding to buoyancy and magnetic pumping. We note that, as recognized in FC2014, the fields permissible at very high field strengths can only be on very small scales. For fields of $10^{7} \mathrm{G}$, still somewhat smaller than the strongest fields considered in FC2014, only flux tubes with $a \leq 2 \times 10^{4} \mathrm{~cm}$ are allowed; for $B=10^{6} \mathrm{G}, a_{\max } \sim 2 \times 10^{6} \mathrm{~cm}$. It is also reassuring to see that large-scale fields (with sizes of order the stellar radii) are permissible at field strengths $10^{4} \mathrm{G}$, since fields of roughly this size and strength have been found in numerical simulations of low-mass stellar dynamos (e.g., Browning 2008). It is important to note that in any realistic dynamo, many scales will likely be present; in this case, these estimates constrain the maximum average size of the field, but do not preclude some fields on both larger and smaller spatial scales.

\subsection{Complicating factors: stable cores, rotation, shear}

We briefly note here a few factors that would influence our conclusions in this section to some degree. We consider the possible presence of a stably stratified core, the existence of strong internal shear, and the influence of rotation on buoyant rise times.

We have so far considered only stars that are convective throughout their interiors; we will largely defer a detailed examination of how these constraints scale in stars possessing radiative cores to future work. But it is worth commenting on a few qualitative features that arise when a stably stratified region is present, whether that region is the result of normal stellar evolution (as in somewhat more massive stars) or instead arises from the stabilizing action of extraordinarily strong magnetic fields (as envi- 
sioned by Mullan \& MacDonald 2001). The presence of a small, stably stratified core slows but generally does not entirely stop the action of the buoyancy instabilities outlined above. There is now the possibility of stable fields, as examined briefly in Mullan \& MacDonald (2001); however, these must still satisfy the criteria outlined in equation 5 or equation 6. Whether strong fields are stabilizing or de-stabilizing depends on the mechanical and thermal properties of the flux tube, but it is generally not possible to avoid instability forever at all wavelengths. The rise times through stably stratified layers in general tend to be much longer than through unstably stratified ones, because they are partly controlled by the slow rate of radiative diffusion into the flux tube (see, e.g., MacGregor \& Cassinelli 2003). But if the stably stratified region is small and only weakly stratified, as it would likely be in the MM01 "magnetic stabilization" scenario, then a number of other effects might well be just as significant as magnetic buoyancy in bringing fields towards the surface. For example, overshooting from the overlying convection into the putative core, and consequent entrainment, would act to mix fields between the two regions; meridional circulations and magnetic diffusion, though much slower, would also tend to link the two zones. Other instabilities not considered here (e.g., the Tayler instability, long studied in the context of massive stars - see, e.g., Spruit 2002; Braithwaite 2006; Zahn et al. 2007) would also come into play at some level. Some of these mechanisms are quite slow compared to buoyancy. But in contrast to the case where the fields are taken to be built self-consistently amidst the convection, magnetism of this strength in a radiative layer is usually expected only to decay: so even if the timescale for instability is longer, its end effect as a loss mechanism for the field is still likely to be severe. We defer discussion of any possible Tayler-Spruit dynamo action (Spruit 2002) in such regions, and likewise of dynamos driven by buoyancy and shear alone (Cline et al. 2003) to later work.

The presence of strong internal shear could also influence the generation of fields, but would not greatly modify our conclusions here. It is plausible that shear might play as great a role as convection in building the fields, and lead to more rapid amplifications of toroidal field than would otherwise be possible. Indeed, although estimates of internal shear in M-dwarfs are highly uncertain, even very small fractions of the overall rotational kinetic energy could, if converted into differential rotation, act more effectively than the convection to build toroidal fields. For example, if an M-dwarf rotating at $10 \mathrm{~km} \mathrm{~s}^{-1}$ managed to sustain internal shear with $\Delta \Omega / \Omega \sim 10^{-3}$, this shear would possess an energy density comparable to (in fact somewhat greater than) that in the convection. Although we would generally expect internal shear to be small in the cases investigated here, owing in part to the effects of the strong Maxwell stresses associated with the magnetism, such proportionally tiny amounts of shear cannot reliably be ruled out. (MacDonald \& Mullan 2015 have investigated a more extreme version of this scenario, considering equipartition of the magnetism with the overall rotational kinetic energy as one limit. It would, however, be difficult to reconcile such equipartition with the simultaneous requirement that the overall angular momentum of the system be conserved.) But rapid amplification by strong internal shear flow is not a panacea: as noted above, no purely toroidal flow can act as a dynamo (Bullard \& Gellman 1954). So although energetically the differential rotation might allow for the build up of strong fields, the timescale on which this can occur must still be limited by the conversion from toroidal back to poloidal field, which is likely still to be accomplished by processes no faster than the convective overturning time. Thus for the dynamo to reach a steady state, the convection still must act to rebuild the fields more rapidly than buoyancy acts to remove them.

Rotation also slows and modifies, but typically cannot stop, the rise of buoyant magnetic flux. The influence of rotation on the buoyancy instability of a continuous field distribution has been studied extensively (e.g., Gilman 1970a; Acheson \& Gibbons 1978; Acheson 1979; Schmitt \& Rosner 1983; Hughes 1985), as has the influence of shear (e.g., Tobias \& Hughes 2004; Vasil \& Brummell 2009). Rotation tends to be somewhat stabilizing, in the sense that though in most cases it does not dramatically modify the onset of instability of a plane layer of field, it does reduce the growth rates of the instability (e.g., Acheson 1979); however, these effects are most significant only when the Alfven speed is much less than $\sim \Omega H$, and still generally allow instability to nonaxisymmetric modes even when rotation is quite rapid. Even adopting a growth rate for such instabilities typical of the rotationally-stabilized problem $\left(\sim v_{a}^{2} /\left(\Omega H^{2}\right)\right.$, with $H$ the scale height and $\Omega$ the frame rotation rate) would, for the field regime examined here, typically result in growth times that remain comfortably shorter than the other relevant timescales in the problem (specifically, both the convective overturning time and the buoyant rise time for small-scale fields). The impact of rotation on rising flux tubes has likewise been studied by many authors (e.g., Choudhuri \& Gilman 1987; Choudhuri 1989; D'Silva \& Choudhuri 1993), with particular focus on the role that Coriolis forces play in setting the emergence latitudes and tilt angles of emerging flux ropes. These latitudes, and the systematic tilt of active regions observed in the Sun (Joy's law), are thought to constitute major observational constraints on the operation of the solar dynamo (e.g., Ossendrijver 2003). Qualitatively, if Coriolis forces are strong relative to buoyancy, rising flux tubes tend to move parallel to the rotation axis; if they are weak, they do not greatly alter the emergence latitudes of the tubes or produce systematic tilt angles akin to Joy's law; intermediate between these two regimes, the rising tubes are not deflected too poleward but still have tilt angles similar to those observed in the Sun. For the extremely strong fields that are the primary focus of this paper, we do not expect Coriolis forces to radically alter the buoyant rise time or the resulting limits on field strength and morphology. In $\S 3$, we provide some more quantitative assessments of the likely effect of rotation on the rise times of thin flux tubes in low-mass stars, using numerical simulations within the thin flux tube approximation.

\section{ASSESSING FIELD EVOLUTION USING SIMULATIONS}

Our analytical estimates of the buoyant rise of thin flux tubes in $\S 2$ are fairly simplistic, as they assume an unchanging tube rising passively under the influence of 
buoyancy. In reality, even if a thin flux tube is produced in the star, it will evolve as it rises under the action of magnetic buoyancy, and in general we expect this to alter its rise time somewhat. We aim in this section to assess whether the broad conclusions from our earlier analytical estimates are in keeping with somewhat more sophisticated models: namely, whether thin flux tubes always rise more slowly than large ones, and whether the rise time is of the same order as our earlier estimates.

\subsection{Computational approach}

To numerically model the rise of isolated, fibril magnetic fields through a 0.3 solar mass star, we adopt the thin flux tube approximation (e.g. Defouw 1976; Roberts \& Webb 1978; Spruit 1981a; Choudhuri \& Gilman 1987). The thin flux tube approximation describes the $1 \mathrm{D}$ reduced MHD equations along the flux tube axis subject to magnetic tension, buoyancy, Coriolis force, and the drag force, with the equations we solve discussed in detail in Fan et al. (1993). The flux tubes we study here rise through a quiescent convective envelope with thermodynamic and stratification quantities taken from a $1 \mathrm{D}$ model of a 0.3 solar-mass M-dwarf as described above.

Following Weber et al. (2011), our simulations start with toroidal magnetic flux rings in mechanical force equilibrium and neutral buoyancy. As in other thin flux tube models, each simulation explicitly considers only a single isolated tube. To ensure initial neutral buoyancy, the internal temperature of the flux tube is reduced compared to the external temperature. There has been some debate regarding the proper initial conditions for thin flux tube simulations, with mechanical equilibrium generally favored over temperature balance in the solar context (see e.g. Caligari et al. 1998). In order to facilitate a magnetic buoyancy instability, the flux tube is perturbed with small undular motions consisting of a superposition of Fourier modes with azimuthal order from $m=0$ to $m=8$ with random phase relations. These perturbations mimic those that would be provided by convective flows, however they are much smaller in amplitude, on the order of a few $\mathrm{cm} \mathrm{s}^{-1}$ in the radial direction. Note that in general, instability need not occur first for $\mathrm{m}=0$ (axisymmetric) modes, so portions of the flux tube may begin to rise before others. Each flux tube evolves adiabatically and is initiated with a latitude of $10^{\circ}$ above the equator at a radial distance of $r_{0}=0.5 R$ (see Tube $\mathrm{A}$ in Fig. 6).

A constraint of the thin flux tube approximation requires that $a_{0} / H_{p} \leq 0.1$ in the region where the flux tube is initiated. At $0.5 R, H_{p}=3.5 \times 10^{9} \mathrm{~cm}$ in our stellar structure model. Therefore, we cannot perform simulations where $a_{0}$ is in excess of $10^{8} \mathrm{~cm}$. Furthermore, the ratio of $a_{0} / H_{p}$ ought to remain small throughout the entire computational domain, ideally less than 1-2. As a result, we stop our simulations once the flux tube apex has reached $0.95 R$ (see Tube $C$ in Fig. 6), operating under the assumption that the rise time through the remaining $0.05 R$ is negligible compared to the total rise. In our simulations, flux tubes of small cross-sectional radius $a_{0} \leq 10^{4} \mathrm{~cm}$ as well as most flux tubes with $B_{0}=10^{4} \mathrm{G}$ suffer from a scenario where the magnetic field strength at the flux tube apex either drops to zero, or weakens drastically in the upper convection zone to a few hundred G, most likely unable to survive the remaining $0.05 R$. Such a scenario is discussed in Moreno-Insertis et al. (1995) in the solar context, where it shown that flux tubes of near equipartition field strengths with small cross-sectional radii exhibit a sudden catastrophic expansion and weakening of the magnetic field at the flux tube apex. For these reasons, we limit our study in this Section to flux tubes of $B_{0}=10^{5}-10^{7} \mathrm{G}$ with $a_{0}=10^{5}-10^{8} \mathrm{~cm}$. The magnetic flux of each flux tube remains constant such that $\Phi=B\left(\pi a^{2}\right)$, resulting in flux values ranging from $3.14 \times 10^{15} \mathrm{Mx}-3.14 \times 10^{23} \mathrm{Mx}$ for our choice of $B_{0}$ and $a_{0}$. In addition, the simulations are also carried out for stellar rotation rates of $1,3,10$, and 40 times that of the Sun, with $\Omega_{\odot}=2.7 \times 10^{-6} \operatorname{rad~s}^{-1}$.

\subsection{Rise times}

In Figure [5] we plot (for models with $B_{0}=10^{6}-10^{7}$ $\mathrm{G}$ ) the total time elapsed from the beginning of the simulation to a time when some portion of the flux tube has reached the simulation upper boundary. For each particular $B_{0}$, in accord with the analytical estimates of Section 2 , the rise time tends to increase as the cross-sectional radius $a_{0}$ decreases. This is shown most prominently in Figure 5 for a rotation rate of $1 \Omega_{\odot}$, and for $10^{7} \mathrm{G}$ flux tubes at all rotation rates considered. Increasing the rotation rate also increases the rise time by suppressing the growth rate of the magnetic buoyancy instability (see e.g. Gilman 1970b; Schüssler et al. 1996).

Rapid rotation also reduces the variation in rise times, effectively normalizing it for smaller $B_{0}$. The Coriolis force increases in magnitude relative to the buoyancy and drag forces as the rotation rate is increased, overwhelming the contribution to the rise time from the size of the tube cross-sectional radius. Furthermore, the increasing magnitude of the Coriolis force relative to the buoyancy force results in a poleward deflection of the rising loop (in the rapidly rotating context, see e.g. Schüssler \& Solanki 1992; DeLuca et al. 1997). An example of this scenario is depicted in Figure 6 for a $B_{0}=10^{6} \mathrm{G}, a_{0}=10^{8} \mathrm{~cm}$ flux tube rotating at $10 \Omega \odot$. A non-axisymmetric $\mathrm{m}=1$ unstable mode develops due to the small amplitude perturbations applied to the initially stable tube. As the flux tube apex rises, conservation of angular momentum drives a counter-rotating flow of plasma elements along the tube which in turn induces an inward directed (toward the rotation axis) Coriolis force. This opposes the outward directed (away from rotation axis) component of the buoyancy force. However, the Coriolis force cannot balance the poleward component of the buoyancy force, and the apex subsequently rises parallel to the rotation axis. The degree to which the tube will be deflected from radial motion depends on the relative magnitude of the Coriolis force to the buoyancy force. In Figure 6. the apex reaches $47^{\circ}$ at the simulation upper boundary. For comparison, given the initial position of our simulated flux tubes, truly parallel motion to the rotation axis results in an emergence latitude at $0.95 R$ of $\sim 58^{\circ}$. Poleward deflection of the rising tube also increases the distance the apex must traverse to reach the surface, thereby increasing the rise time as well. Flux tubes of $10^{7} \mathrm{G}$ only begin to show moderate deflection of $\sim 10^{\circ}$ poleward at $40 \Omega_{\odot}$ for the thickest tube $\left(a_{0}=10^{8}\right.$ 


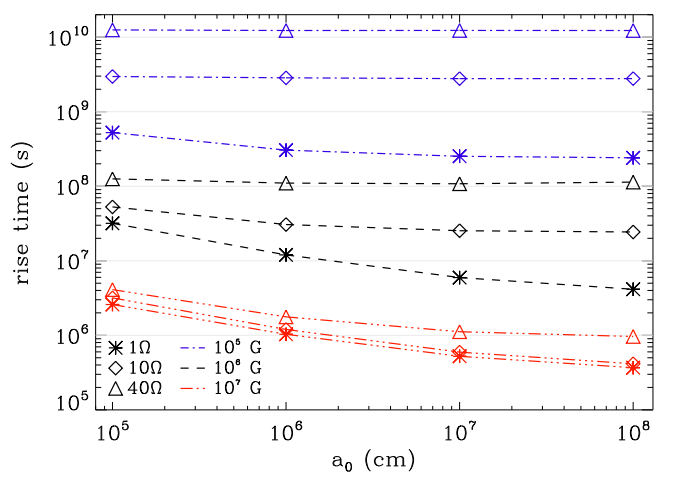

Figure 5. Rise times in thin flux tube simulations as a function of initial cross-sectional radius $a_{0}$ for various $B_{0}$ and $\Omega_{0}$. For each particular $B_{0}$, the rise time tends to increase as $a_{0}$ decreases. However, rapid rotation normalizes this variation especially for smaller $B_{0}$.

$\mathrm{cm})$. Due to their large magnetic field strength, the magnitude of the Coriolis force is always much less than the buoyancy force, even at high rotation rates.

The simulated rise times in Figure [5] are fairly close to the analytical estimates in Section 2. For example, for extreme fields of $10^{7} \mathrm{G}$, Equation (3) gives a rise time of about $8 \times 10^{6} \mathrm{~s}$ for a flux tube of $a_{0}=10^{5} \mathrm{~cm}$. In our simulations, a flux tube with those parameters rises about a factor of two faster than this if $\Omega$ is 40 times the solar rate, and a factor of 3-4 faster if $\Omega / \Omega_{\odot}$ is between 1 and 10. Placing $r_{0}$ at $0.25 R$ or the origin would increase the rise times given in Figure 5 somewhat. A smaller $r_{0}$ also increases the radius of curvature of the flux tube, thereby increasing the magnetic tension and decelerating the buoyant rise of the flux tube apex. Including the effects of convective flows and radiative diffusion on the motion of thin flux tubes in our simulations will also have an impact on rise times; we expect this would be minimal for $10^{7} \mathrm{G}$ flux tubes owing to their extreme buoyancy, but could be more pronounced at lower field strengths. Though clearly these differences would affect our maximum field estimates in Section 2 and Section 3, the difference appears to us to be small in comparison to the rather large theoretical uncertainties that underlie this whole subject. We defer further discussion of the dynamics of rising flux tubes in fully convective stars, and their emergent properties at the surface, to later work.

\section{OHMIC DISSIPATION AND HEATING}

Though the interiors of low-mass stars are highly ionized, they are not perfect conductors. The currents sustained in the plasma must therefore undergo Ohmic dissipation, with an associated heating (energy per unit time per unit volume) $=j^{2} / \sigma=4 \pi \eta j^{2} / c^{2}$. Here $\eta$ is the magnetic diffusivity (with units of $\mathrm{cm}^{2} \mathrm{~s}^{-1}$ ), related to the conductivity of the medium by $\eta=c^{2} /(4 \pi \sigma)$. Recall that the current density $\mathbf{j}=(c / 4 \pi)(\boldsymbol{\nabla} \times \mathbf{B})$.

In other contexts, several authors have noted that for some magnetic field strengths and morphologies, this Ohmic dissipation may represent a significant heating source. For example, Liu et al. (2008) argued that this provided a powerful constraint on the depth of zonal winds in Jupiter and Saturn: If the winds extend into the interior, they would tend to stretch the observed surface poloidal fields into interior azimuthal fields; if

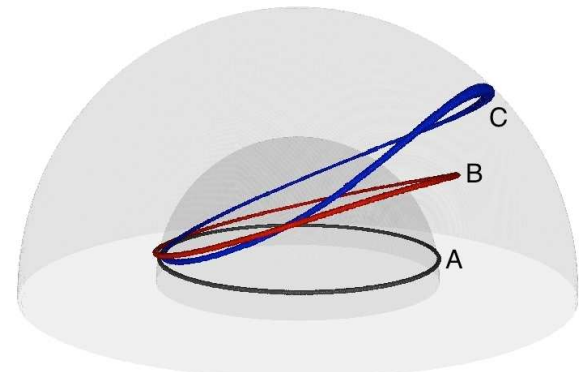

Figure 6. Depiction of a $B_{0}=10^{6} \mathrm{G}, a_{0}=10^{8} \mathrm{~cm}$ flux tube rotating at $10 \Omega_{0}$ for snapshots at three different times in its evolution. Tube A shows the initial position of the flux tube at $r_{0}=0.5 R$, with a darker inner hemisphere mapping out a surface of radius $r_{0}$. Tube $\mathrm{B}$ and Tube $\mathrm{C}$ have apices that reach $0.75 R$ and $0.95 R$, respectively. The simulation upper boundary at $0.95 R$ is shown by the outer gray hemisphere. Rapid rotation forces the deflection of the tube apex to $47^{\circ}$. Each tube segment has been given a 3D extent according to the local cross-sectional radius, and the tube evolution is shown in a reference frame co-rotating with the star.

the winds extend too deeply, the heating associated with the dissipation of these fields could in some cases exceed the planet's luminosity. Later, Batygin \& Stevenson (2010) invoked Ohmic dissipation as a mechanism for inflating close-in extrasolar planets: assuming reasonable profiles for the zonal winds and surface magnetism of these objects, they argued that the associated Ohmic heating could be large enough (and deposited deep enough) to lead to anomalously large radii. Several other papers have investigated variants of this scenario in some detail, assuming different models for the winds, conductivities, and magnetic fields of these objects (e.g., Huang \& Cumming 2012; Wu \& Lithwick 2013) and more recently simulating the atmospheric winds and magnetic fields with varying levels of sophistication (Rauscher \& Menou 2013; Rogers \& Showman 2014; Rogers \& Komacek 2014).

In this section, we investigate the Ohmic dissipation associated with the field strengths and morphologies examined in $\S 2$. We give general estimates of the current densities associated with these fields, and briefly note a few firm limits on the currents associated with any field distribution that is actively maintained against decay. Employing plausible conductivity profiles for the interiors of these objects, we then calculate the power from Ohmic dissipation for fields of varying strength and geometry. Some combinations of field strength and topology lead to very large rates of Ohmic heating, and we argue that this sets a complementary limit on the strength of fields in the interiors of low-mass stars.

\subsection{Estimates and limits of current density}

The current density $j$ depends on both the strength and spatial scale of the magnetism. To order of magnitude, for a field varying on spatial scale $a, j \sim c B / a$, so for fixed total field strength, smaller-scale fields are associated with stronger currents. We will generally employ this simple estimate for our calculations below.

Before applying this estimate, we note that firm lower bounds can be derived for the current density, but these will tend to underestimate the magnitude of $j$ for smallscale fields. Intuitively, it seems clear that the current cannot be less than $j \sim c B / R$ with $R$ the radius of the star, and it is straightforward to show that this is 
the case. More specifically, for divergence-free fields confined in a sphere of radius $R$ and matching to a decaying potential outside that sphere, one can prove that

$$
\int|\nabla \times \mathbf{B}|^{2} d V \geq \frac{\pi^{2}}{R^{2}} \int|B|^{2} d V
$$

(e.g., Jones 2008). Another possible constraint comes from consideration of the induction and momentum equation: the rate of change of magnetic energy is related to the Poynting flux out of the volume and to the work done by the fluid $(\propto \mathbf{j} \cdot \mathbf{v} \times \mathbf{B}=-\mathbf{v} \cdot \mathbf{j} \times \mathbf{B})$, in principle providing a constraint on the minimum $j$ for a given rate of induction. (To see this, note that $\mathbf{j} \cdot \mathbf{v} \times \mathbf{B}$ is bounded by the product of $u_{\max }$ and the square root of volume integrals over $j^{2}$ and $B^{2}$; see, e.g., Childress 1969 , where a similar line of reasoning is used to provide a bound on the minimum magnetic Reynolds number needed for dynamo growth.) Related arguments have also been used in an effort to infer, for example, the Ohmic dissipation in Earth's core (e.g., Roberts et al. 2003). In practice these estimates typically provide only lower bounds, and we have not found them to be particularly useful for constraining the current density except in special circumstances. Ultimately, any realistic field distribution will likely possess fields over a range of spatial scales, but it is always possible to find a characteristic $a$ such that the current density, averaged over some volume, is $j \sim c B /(4 \pi a)$. This need not, and in general will not, correspond to the smallest or largest scales physically present in the system.

\subsection{Conductivity profiles}

For specificity, we primarily consider the interior of a 0.3 solar mass M-dwarf, whose temperature, density, and pressure are as in previous sections given by a 1-D stellar model (Chabrier \& Baraffe 1997). These models calculate the conductivity using the methods of Potekhin (1999); Potekhin et al. (1999). Although initially developed for neutron star envelopes and white dwarf cores, these recover previous calculations (Hubbard \& Lampe 1969; Itoh et al. 1983; Mitake et al. 1984; Brassard \& Fontaine 1994) at lower densities and cover the range of temperatures and densities characteristic of low-mass stars and brown dwarf interiors. They have been used for instance in Chabrier et al. (2000) to calculate the conductive opacities in old and massive brown dwarfs. They are presently the most detailed and accurate conductivity calculations for conditions ranging from neutron stars to jovian planets. The conductivity profile is shown as the solid line in Figure 7. In the regime considered here, the conductivity can be taken to be independent of the magnetic field strength and morphology.

For ease of comparison with other work, we note that the conductivity profile employed here is not too different from that derived by other methods. A basic rule of thumb for electron conduction is that the magnetic diffusivity (in $\mathrm{cm}^{2} \mathrm{~s}^{-1}$ ) is $10^{4} T^{-1.5}$, with $T$ in millions of K. Huang \& Cumming (2012) also give formulae for conductivity in partially degenerate matter, and we quote these here for convenience. In general we have $\sigma=n_{e} e^{2} / m_{e} \nu$, with $\nu$ the collision frequency and $n_{e}$ the electron number density. If the plasma is ion-

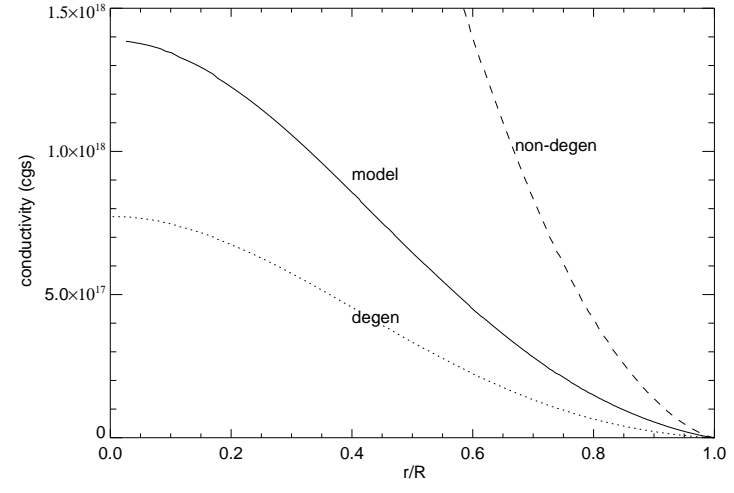

Figure 7. Estimates of the radial variation of conductivity in the interior of a low-mass star (in cgs units). Shown are the values adopted in this work (solid line), together with estimates for purely degenerate and non-degenerate matter (see text).

ized enough for electron-proton collisions to dominate, $\nu \approx 4 e^{4} m_{e} \Lambda /\left(3 \pi \hbar^{2}\right) \approx 1.8 \times 10^{16} \mathrm{~s}^{-1}$ in the fully degenerate limit, or $\nu \approx 6.4 \times 10^{23} \mathrm{~s}^{-1} \rho Y_{e} T^{-3 / 2}$ if not fully degenerate. Purely for comparison purposes, we show both the fully degenerate and non-degenerate conductivity curves in Figure 7 as well (dashed and dotted lines). The conductivity profile adopted here is intermediate between these two extremes.

A typical value of $\sigma$ in the deep interior (namely, the mean value over the inner $1.5 \times 10^{9} \mathrm{~cm}$ ) is about $8 \times 10^{17}$ (cgs), implying a magnetic diffusivity of about $80 \mathrm{~cm}^{2}$ $\mathrm{s}^{-1}$. The "rule of thumb" calculation above gives similar values. Assuming the plasma is fully degenerate would result in a typical conductivity about 0.16 times the nondegenerate value, or equivalently diffusivities that are about a factor of 6 larger.

\subsection{Ohmic dissipation for proposed field distributions}

The energy per unit volume per unit time from Ohmic dissipation is given by the square of the current density divided by the conductivity. The total power associated with Ohmic dissipation in a volume extending from the origin to radius $R$ is

$$
L_{\mathrm{dissip}}=\int_{0}^{R} 4 \pi r^{2} \frac{j(r)^{2}}{\sigma(r)} d r
$$

where all symbols take their usual meanings. For any given field distribution and characteristic scale $a$, it is then straightforward to calculate the total Ohmic dissipation.

In this section we briefly investigate the dissipation associated with some of the strong field distributions examined in previous work (FC2014, MM). These published models are characterized only by a characteristic field strength at every depth; the morphology of the field is left unspecified. Clearly the characteristic spatial size of the field $a$ has a significant impact on the dissipation: the total power from dissipation scales like $(B / a)^{2}$, so largescale fields lose less energy (per unit time) to dissipation than small-scale ones.

In one of the models considered extensively in FC2014 (their "Gaussian" model), the magnetic field strength is assumed to vary with radius as

$$
B_{\mathrm{FC} 14}=B_{\max } e^{-0.5\left(\left(r_{t}-r\right) / \sigma\right)^{2}}
$$




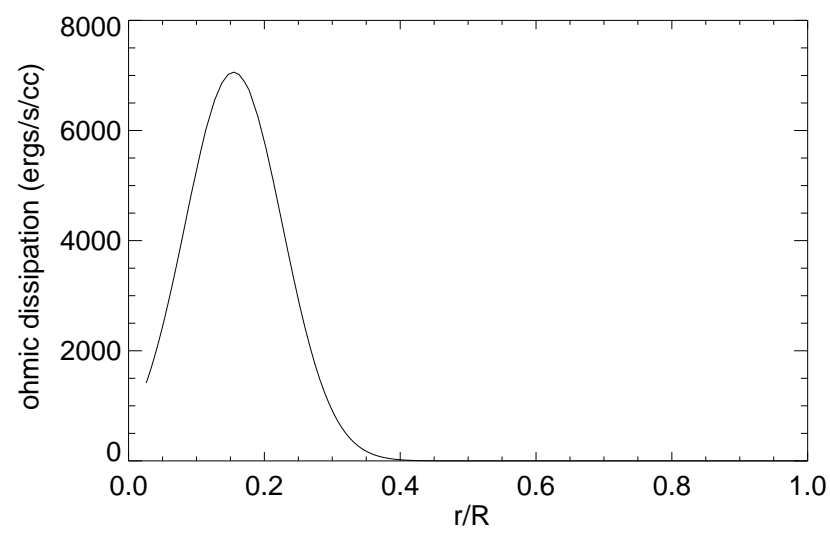

Figure 8. Heating from Ohmic dissipation (energy per unit volume per unit time) dissipated for the FC2014 field if $a=10^{6} \mathrm{~cm}$ and the conductivity profile is as assumed above.

where $r_{t}$ and $\sigma$ are 0.15 and 0.10 times the maximum radius respectively, and $B_{\max }=4.0 \times 10^{7} \mathrm{G}$. We assume the simple model for current density above $(j \sim c B / 4 \pi a)$, and consider three representative values of $a$, namely $10^{6}, 10^{7}$, and $10^{8} \mathrm{~cm}$. It is worth noting that at this extreme field strength, even these (comparatively small scale) fields would not actually be stable against magnetic buoyancy according to the most stringent of our limits above; if the field were composed entirely of thin flux tubes, they would need to have a typical radius $a$ below $10^{5} \mathrm{~cm}$ (which would make $j^{2}$ about 100 times larger than our largest estimates here) to be stable.

Figure 8 considers the Ohmic dissipation at each radius for this model, assuming the conductivity is given by the model above, and Figure 9 calculates the integrated power from Ohmic dissipation ( $L_{\text {dissip }}$ above), with the total luminosity of the star overplotted as a horizontal dashed line. It is clear that the combination of very strong fields (as required in the FC2014 model to explain inflated radii) and fairly small characteristic lengthscales would result in Ohmic dissipation exceeding the luminosity of the entire star. Larger values of $a$ lead to less dissipation, with the heating scaling as $a^{-2}$. Thus, while adopting the FC2014 peak field strength and values of $a=10^{6} \mathrm{~cm}$ leads to dissipative luminosities that vastly exceed the stellar luminosity, and taking $a=10^{7} \mathrm{~cm}$ $\mathrm{cm}$ yields dissipation of the same order as $L_{*}$, values of $a \geq 10^{8} \mathrm{~cm}$ at the same field strength would lead to fairly negligible heating.

\subsection{Minimum spatial scales consistent with Ohmic constraints}

The 1-D stellar models in general use today include no explicit allowance for Ohmic heating in the interior, so situations in which the integrated Ohmic dissipation approaches the stellar luminosity represent a possible contradiction. We can therefore use the constraint that the total dissipation not exceed $f L$, where $f$ is a factor less than 1 , to constrain the current density within the star. Furthermore, although the total dissipation in some convective systems may exceed the total luminosity without violating the laws of thermodynamics (see Hewitt et al. 1975, and explicit numerical examples in Jones \& Kuzanyan 2009; Viallet et al. 2013), this is only

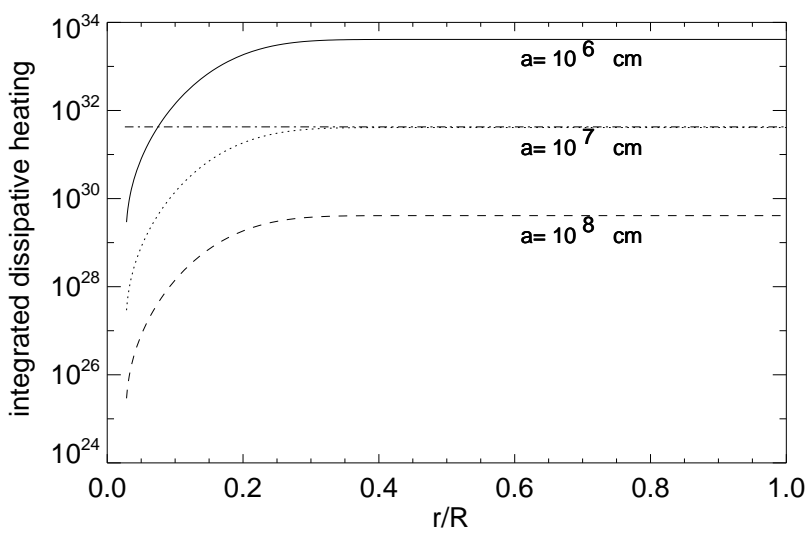

Figure 9. Total integrated luminosity from Ohmic dissipation in the same model as a function of radius, for three different values of characteristic dissipative scale $a$, and compared to the total stellar luminosity (shown as dashed line).

the case when the (thermal) stratification is strong. For the gradually-declining temperatures in the interior of a low-mass star, $f$ must be below unity. (Formally, in many cases it is bounded by a value of order $r / H_{t}$, with $H_{t}$ the thermal scale height, which in turn implies $f$ less than unity for the deepest portion of the interior.) For a given profile of $B$ (as in the FC2014 profiles considered above), and assuming the current density is related by the simple scaling relations above to the characteristic spatial scale of the field, we can then derive a minimum characteristic scale for the field. Smaller-scale fields (at fixed overall field strength) would result in too much dissipation. The resulting estimate is

$$
a_{\min }=\left[\frac{\int_{0}^{R} 4 \pi r^{2}\left(\frac{c B(r)}{4 \pi}\right)^{2} \frac{1}{\sigma(r)} d r}{f L}\right]^{1 / 2}
$$

We calculate $a_{\mathrm{min}}$ for specific field distributions from FC2014 below. First, though, we note a few cases in which this equation has a particularly simple interpretation. If the dissipation $j^{2} / \sigma$ does not depend on position within the star, then it is straightforward to show that our expression for $a_{\min }$ is equivalent to

$$
a_{\min }^{2} \sim \bar{\eta} R^{3} \frac{1}{f L} \bar{B}^{2},
$$

where $\bar{\eta}$ is the magnetic diffusivity averaged over the volume and $\bar{B}$ is the average field strength. This criterion is more transparently written as

$$
\frac{E_{\mathrm{mag}}}{\tau_{\eta}\left(a_{\mathrm{min}}\right)} \lesssim f L
$$

where $E_{\text {mag }}=\left(4 \pi R^{3} / 3\right)\left(B^{2} / 8 \pi\right)$ is the total magnetic energy in the volume and $\tau_{\eta}\left(a_{\mathrm{min}}\right)$ is the magnetic diffusion time for fields on scale $a_{\mathrm{min}}$. If $a$ is reduced below $a_{\text {min }}$, the characteristic diffusion time becomes shorter, so the power dissipated per unit time is larger; we require that it be smaller than the luminosity of the star (or if $f \neq 1$, some fraction thereof).

For the specific 1-D stellar model considered here, with average Ohmic diffusivities as quoted above, this requirement translates to a linear relationship between $a_{\text {min }}$ and 
the average field $B$, namely $a_{\min } \approx \sqrt{6 / f} \bar{B} \mathrm{~cm} \mathrm{G}^{-1}$, where the total power from Ohmic dissipation is not to exceed $f L$. Although values of $f$ approaching or exceeding unity are possible in some cases, we will take $f=0.4$ as a reasonable limit for the deep interior: above this, we expect the structure of the star would be significantly affected by the Ohmic dissipation, leading to changes that are at least as great as those purportedly arising from modifications to the convective heat transport. For this value of $f, a_{\min } \approx 3.9 B \mathrm{~cm} \mathrm{G}^{-1}$, so values of $B \approx 10^{6}$ $\mathrm{G}$ require that the field be structured predominantly on scales larger than about $4 \times 10^{6} \mathrm{~cm}$.

The crude assumption that $j^{2} / \sigma$ is independent of depth is not as unrealistic as it might at first appear. Suppose the field strength at every depth is a fixed multiple of the equipartition field strength, $B \propto \sqrt{(} \rho) v_{c}$, and that the convective velocity does not vary too much with depth, so $B^{2} \propto \rho$. The density and temperature structure of a fully convective star is reasonably well approximated by a polytrope with index $n=1.5$, in which case the density and temperature follow relations of the form $\rho=\rho_{c}(\theta(\xi))^{1.5}, T=T_{c} \theta(\xi)$, with $\xi$ a dimensionless radius $r=r_{n} \xi$ (in turn involving a scale length $\left.r_{n}^{2}=(n+1) P_{c} /\left(4 \pi G \rho_{c}^{2}\right)\right)$. Recall that the conductivity in the non-degenerate case is $\sigma \propto T^{3 / 2}$, which in the $\mathrm{n}=1.5$ polytropic case implies $\sigma(r) \propto \theta^{3 / 2} \propto \rho(r)$. Hence for the scaled-equipartition field, the ratio $B^{2} / \sigma \approx \rho(r) / \rho(r)$ is approximately constant.

It is worth reiterating that for more realistic field distributions containing a range of spatial scales, with $B$ in general a function of spatial scale, one could still define an $a_{\text {min }}$ consistent with the requirement that Ohmic dissipation not exceed a given luminosity. In general this estimate would correspond neither to the largest scales present in the system nor the smallest, but to a characteristic scale intermediate between the two. For the conductivity profiles and field strengths considered here, the microphysical dissipation scale, taken as the scale $l$ at which the magnetic Reynolds number $R m=u(l) l / \eta \approx 1$, is much smaller than $a_{\text {min }}$ for any plausible variation of $u$ with length $l$ : e.g., for $u(l)=u(R)(l / R)^{\alpha}$ as before, the dissipative scale is given by

$$
l_{\eta} \approx\left(\frac{\eta R^{\alpha}}{u(R)}\right)^{\frac{1}{1+\alpha}} .
$$

If $\alpha=1 / 3$, this reduces to the familiar rule that $l_{\eta} \propto$ $R m^{-3 / 4}$, whereas if $\alpha=1$ it is equivalent to the statement that the dissipation time at $l_{\eta}$ is equal to the largescale dynamical time $R / u(R)$. In both cases $l_{\eta} \ll a_{\text {min }}$.

In what follows, we will generally employ both the simplest estimate of $a_{\text {min }}$ above (i.e., assuming $j^{2} / \sigma$ is approximately constant) and a slightly more complex calculation in which we have numerically integrated equation 24 for specific field profiles from FC2014 (assuming the conductivity is given by the 1-D model as above). Both estimates of $a_{\text {min }}$ scale in the same way with $B$, but they differ by constant factors of order unity. In both cases, because $a_{\min }$ increases with $B$, conflict with the requirements of $\S 2$ that the field be larger than some given scale to avoid rapid losses due to magnetic buoyancy instability, are in principle possible. We explore this conflict in the next section.

\section{COMBINED LIMITS ON FIELD STRENGTH}

We have so far highlighted a few of the difficulties involved in finding extremely strong magnetic field configurations that could persist for indefinite intervals. In $\S 2$ we reviewed a variety of work on magnetic buoyancy instabilities in stellar interiors. We have not analyzed arbitrarily complex field distributions, but have considered two extreme cases: isolated flux tubes initially in equilibrium with their field-free surroundings, and smooth spatially-varying distributions of magnetism. Both the strength of the field and its spatial structure modify the growth of these instabilities. Fields as strong as those considered in some models are likely only stable against buoyancy (or have buoyant rise times shorter than plausible regeneration timescales for the magnetism) if the field is predominantly structured on very small scales. An equivalent statement is that at any fixed field strength, fields larger than some maximum spatial scale $a_{\max }$ are unstable; smaller-scale fields might conceivably persist. At fields of order the equipartition strength, this maximum allowable spatial scale extends to the largest possible scale in the system (the stellar radius), but at very strong field strengths only small-scale fields are consistent with the constraint of buoyancy.

But associated with the small-scale fields necessitated by buoyancy are intense currents. In $\S 4$ we argued that the Ohmic heating associated with strong, small-scale fields would greatly exceed the stellar luminosity in some cases. Equivalently, because the Ohmic heating associated with a field of magnitude $B$ and average spatial scale $a$ scales with $B / a$, at fixed field strength there is a minimum spatial scale $a_{\text {min }}$ consistent with the assumed stellar luminosity: fields structured predominantly on smaller scales dissipate too much energy.

These constraints can be combined to give an approximate limit on the maximum possible field strength in the stellar interior. In Figure 10 we show the estimates of $\S 2$, which give $a_{\max }$ for a collection of flux tubes at any given field strength $B$, together with the calculation from $\S 4$ of the minimum characteristic scale $a_{\text {min }}$ consistent with the constraints of Ohmic dissipation. The solid lines show the analytical estimates of equations 25] and 20 (employing a typical depth $r=0.25 R$ for the estimate of the rise time), which we take as our most representative calculations. At low field strengths (of order the equipartition value), a wide range of characteristic scales are compatible with both constraints. At higher field strengths the window of allowable field strengths narrows, and eventually the two lines $a_{\min }$ and $a_{\max }$ cross, indicating that no characteristic field scale can simultaneously meet both constraints. We regard this intersection as an upper limit on the achievable field strength: above it, any field that is stable to magnetic buoyancy would result in too much Ohmic heating.

The actual value of $B_{\max }$ naturally depends on what estimates are adopted for $a_{\min }$ and $a_{\max }$, but an upper limit (i.e., an intersection of $a_{\min }$ and $a_{\max }$ ) exists for all the models we have considered here. For the specific model of $a_{\max }$ considered in equation 20, which estimates the maximum field that could be pumped downward against magnetic buoyancy (or, equivalently, the largest field that could be regenerated by slow, largescale eddies), and for the estimate of $a_{\text {min }}$ from equation 
25 (which assumes the dissipation $j^{2} / \sigma$ is independent of depth, and allows Ohmic dissipation up to 40 percent of the stellar luminosity), the resulting maximum field strength is about $800 \mathrm{kG}$. More generous assumptions about the effectiveness with which flows could rebuild magnetism as it is emptied out by magnetic buoyancy, as represented for instance by the "fast eddy/shear" model of field generation and the upper (dotted) $a_{\max }$ line in Figure 10, could lead to larger $B_{\max }$. Adopting the intermediate "cascade" model for field regeneration (with a velocity dependent on scale $l$ as $v \propto l^{\alpha}, 0<\alpha<1$ ) leads to a maximum field estimate intermediate between these two. We suspect that the strictest of these limits is likely to be the most robust, in part because it also reflects a limit on the maximum field that could persist for some time against magnetic buoyancy without ongoing rapid regeneration by dynamo action. (Of course such a field would inexorably decay from Ohmic dissipation, but this process is slow when compared to the timescales associated with magnetic buoyancy or convection.)

For comparison, we have also plotted (as the dasheddot line) $a_{\mathrm{min}}$ calculated for a field whose variation with radius follows the "Gaussian" profile from FC2014 as described above, and which attains a maximum value given by the x-axis. At each point we have numerically calculated the integral over $j(r)^{2} / \sigma(r)$, still assuming that $j(r)=c B(r) /(4 \pi a)$, and allowing $f=1$. The variation with $B$ is the same, but the curve is offset slightly with respect to our simple model (and slightly different value of $f$ ), so the maximum field strength consistent with both buoyancy and dissipation is slightly higher (about $1 \mathrm{MG}$ ). The conclusions remain otherwise the same as in the simpler model.

An explicit expression for $B_{\max }$ follows from setting $a_{\min } \approx a_{\text {max }}$. In the specific case of "slow" eddies represented by the solid line in Figure (10), and taking $H_{p} \approx R$ in the interior as in equation 19, we have that

$$
B_{\max }^{3} \approx B_{\mathrm{eq}}^{2} \sqrt{\frac{f L}{\eta R}}
$$

where as before $B_{\text {eq }}$ is an estimate of the field in equipartition with the convective kinetic energy. The term under the square root can be understood intuitively as the extraordinarily strong field that would satisfy $a_{\min }=R$ : i.e., the field for which the Ohmic dissipation associated with dissipation on the largest possible scale $R$ would still exceed the luminosity of the system,

$$
B_{\eta} \approx \sqrt{\frac{f L}{R \eta}} .
$$

For the model considered here, $B_{\eta}$ is in excess of $10^{9}$ $\mathrm{G}$, and $B_{\text {eq }} \sim 10^{4} \mathrm{G}$, yielding the quoted $B_{\max }$ of order $8 \times 10^{5} \mathrm{G}$.

We emphasize that our estimates here refer to the maximum mean field that could persist, in the same way that our estimates of scale correspond to a single spatial scale that characterizes the overall field distribution. This is convenient for comparison with prior models of the interior magnetism in low-mass stars (e.g., the models of FC2014 and MM), which are likewise characterized by a single field strength at each depth. More realistic

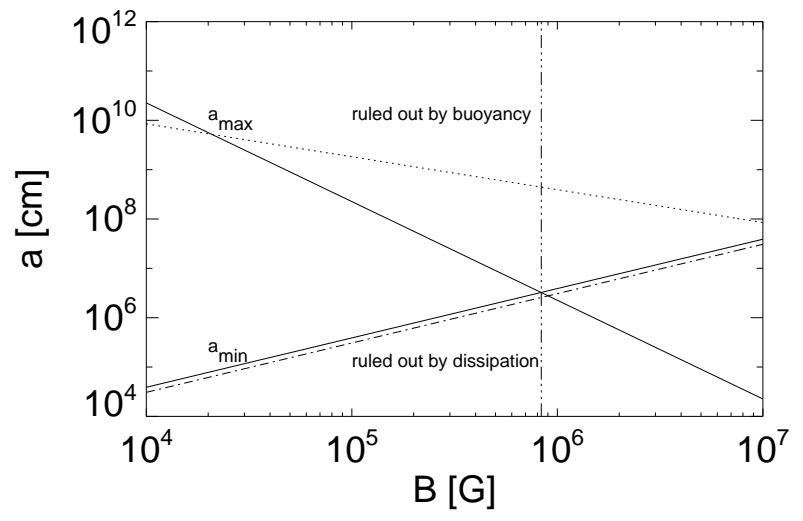

Figure 10. Characteristic spatial scales of magnetism that are ruled out by Ohmic dissipation and magnetic buoyancy. At given field strength, configurations consisting primarily of very large scale fields are ruled out by magnetic buoyancy, while very small-scale fields are inconsistent with the Ohmic dissipation constraint. Beyond a maximum field strength $B_{\text {crit }}$, denoted by a dashed vertical line for a particular choice of models, no spatial scales of the field are consistent with both constraints.

field configurations will of course contain a range of field strengths and morphologies, which may exceed $B_{\max }$ in some locations and contain structures smaller than $a_{\text {min }}$ without violating in a global sense any of the constraints explored in this paper. But it is always possible to define a mean field and a characteristic spatial scale, and these must obey some variant of the constraints noted here.

\section{DISCUSSION: TOWARDS EXTENSIONS TO OTHER MASSES}

Our discussion thus far has concentrated on the constraints provided by buoyancy and dissipation in the interior of a fully convective 0.3 solar-mass M-dwarf. Here, we present a very simple estimate of how these constraints might scale to other masses, while retaining the assumption that the interior of the star is fully convective. As noted above, the extension to cases with a stably stratified core is somewhat more complex, and considerably more uncertain, so we defer it to later work.

We concluded in $\S 5$ that the maximum field strength consistent with the constraints of both Ohmic dissipation and magnetic buoyancy is, to order of magnitude, $B_{\max }^{3} \approx B_{\mathrm{eq}}^{2} B_{\eta}$, with $B_{\eta}=\sqrt{f L /(R \eta)}$ and $B_{\text {eq }}=\sqrt{4 \rho} v_{c}$. We also assume that the convective velocity follows the mixing-length scaling, $v_{c} \propto(L R / M)^{1 / 3}$. Let us further assume a power-law relationship between stellar mass and luminosity, $L \propto M^{\beta}$, and take mass roughly proportional to stellar radius, $R \propto M$. (This latter assumption will break down in degenerate objects, but is reasonable for objects on the hydrogen-burning main sequence.) We further note that for an object composed of ideal gas in hydrostatic equilibrium, the central temperature is roughly constant in this mass regime (e.g., Chabrier \& Baraffe 1997), implying $\eta$ is in turn approximately constant. Then $B_{\eta} \propto \sqrt{M^{\beta} / M}=$ $M^{(\beta-1) / 2}$. The equipartition field strength scales like $B_{\text {eq }} \propto M^{(\beta-3) / 3}$, so some manipulation yields

$$
B_{\max } \propto M^{\frac{7 \beta-15}{18}} .
$$

In 1 -D models (Chabrier \& Baraffe 1997), $\beta \approx 5$ for 
masses between 0.5 and 1 solar masses, and $\beta \approx 2.4$ between 0.1 and 0.5 solar masses. (The eventual flattening of the mass-luminosity relationship in these models is due primarily to the formation of $\mathrm{H}_{2}$ and the onset of convection in the atmosphere, rather than to deep interior properties.) Hence $B_{\max }$ would be expected to vary only weakly with mass in the very low mass star/brown dwarf regime (for $\beta=2.4, B_{\max } \propto M^{0.1}$ ).

\section{CONCLUSIONS AND PERSPECTIVES}

We have aimed to examine here whether the very strong interior magnetic fields invoked in some models of low-mass stars, however initially established, could be maintained indefinitely. For the very simple field configurations considered here, which consist primarily of collections of thin flux tubes, we find that Ohmic dissipation and magnetic buoyancy combine to yield interesting constraints on possible field strengths and morphologies. We argued in $\S 2$ that strong fields might avoid rapid rise from magnetic buoyancy instability, or be regenerated faster than they rise, only if they are structured on very small scales. But the Ohmic dissipation associated with such small-scale magnetism would in some cases exceed the luminosity of the star $(\S 4)$. In $\S 5$ we concluded that above a strength of order $1 \mathrm{MG}$, no field can satisfy both constraints simultaneously, so we regard this as a practical upper limit on the fields that could be maintained.

A principal limitation of our work is its reliance throughout on very simple models of the magnetic field's spatial structure. Our calculations of the rise time of buoyant fields draw on the thin flux tube approximation, which essentially asserts that the field is composed of well-defined tubes characterized only by their strength and cross-sectional area. We argued that structures akin to these tubes might arise, for example, from the breakup of smooth magnetic layers, which are also provably subject to buoyancy instabilities. This approach is not unreasonable, having been used to considerable effect in the solar community for decades, and it has the great advantage that it allows us to estimate quantities like the rise time analytically. But it is still only an approximation. In particular, our assumption that the rise of buoyant flux is impeded essentially by drag, while also in line with many previous authors, is likely a considerably simplified description of a more complex process (see, e.g. Hughes \& Falle 1998). Assessing whether more complex configurations of field are as susceptible to buoyant instability, and likewise the rate at which such fields are really regenerated by dynamo action, is probably not possible without recourse to more sophisticated numerical simulations.

Likewise, our estimates of the Ohmic dissipation assume for simplicity that the field can be characterized by only one spatial scale; in reality, any dynamogenerated field will contain a range of spatial and temporal scales, and the dissipative heating may involve more complex reconnection processes than considered here (e.g., Lazarian \& Vishniac 1999). We have also ignored spatial anisotropies in the magnetism (or the flow), whether induced by rotation or by strong fields themselves, even though these are surely present (see, e.g., Oruba \& Dormv 2014; Davidson 2013). But it is always possible to define a characteristic scale for the field, and for its dissipation, lying intermediate between the largest scales in the system and the smallest. Ultimately we think the dissipation, whatever its distribution with scale, must still obey some form of the constraints examined here.

In other ways, though, our estimates are very conservative. In our estimates of plausible regeneration times, for example, we have adopted an essentially kinematic estimate (taking the growth time for the magnetism to be of order the convective turnover time); in general, one would expect the very strong fields examined here to exert considerable Lorentz feedbacks on the flow, leading in turn to much slower regrowth than estimated here. This would act to lower the maximum sustainable field strength.

Perhaps the most robust conclusion from our work is that any model for the interiors of low-mass stars that invokes very strong magnetic fields must consider not just the strength of those fields but also their morphology. Fields with different spatial distributions behave differently: if the field is mostly on small scales, it might conceivably be regenerated faster than buoyancy instabilities can act to remove it, whereas large scale fields take longer to regenerate and are more susceptible to rapid buoyant losses. On the other hand, fields that vary on small scales are subject to stronger Ohmic dissipation, and we have shown that this presents significant constraints in the most extreme cases.

The full implications of this work for the structure of low-mass stars are not yet clear. Other authors (e.g., FC14) have indicated that magnetic fields of great strength would be required to appreciably inflate the radii of low-mass stars (within the context of a particular mixing-length model for the convective transport); FC14 in particular ultimately concluded that such fields were unlikely for a variety of reasons. Our work partly echoes and complements theirs, by showing explicitly that strong fields on small spatial scales conflict with the combined constraints provided by buoyancy and Ohmic dissipation. Despite the many caveats noted above, we think that the basic constraint provided by buoyancy and dissipation is likely to be robust, and hence that the very strong fields examined in some previous models are not feasible. Assuming that the conflict between observed and predicted radii in these stars is real, this suggests either that other phenomena are acting to "inflate" the stars, or that even weaker magnetism (coupled with rotation) can affect the convective transport. We intend to examine these possibilities in future work.

We thank Isabelle Baraffe for providing structure models used in this paper, and for many helpful discussions. This work was supported by the European Research Council under ERC grant agreements no 337705 (CHASM) and (FP7/2007-2013) no 247060, and by a Consolidated Grant from the UK STFC (ST/J001627/1). We also thank Andrew West, who supported AM's work on this project. Some of the calculations for this paper were performed on the DiRAC Complexity machine, jointly funded by STFC and the Large Facilities Capital Fund of BIS, and the University of Exeter supercomputer, a DiRAC Facility jointly funded by STFC, the Large Facilities Capital Fund of BIS and the University of Exeter. 


\section{REFERENCES}

Abbett, W. P., Beaver, M., Davids, B., et al. 1997, ApJ, 480, 395 Acheson, D. J. 1979, Sol. Phys., 62, 23

Acheson, D. J., \& Gibbons, M. P. 1978, Journal of Fluid Mechanics, 85, 743

Barker, A. J., Silvers, L. J., Proctor, M. R. E., \& Weiss, N. O. 2012, MNRAS, 424, 115

Basri, G., Walkowicz, L. M., \& Reiners, A. 2013, ApJ, 769, 37

Batygin, K., \& Stevenson, D. J. 2010, ApJ, 714, L238

Beresnyak, A., \& Lazarian, A. 2010, ApJ, 722, L110

Biskamp, D., \& Müller, W.-C. 2000, Physics of Plasmas, 7, 4889

Boldyrev, S. 2006, Physical Review Letters, 96, 115002

Braithwaite, J. 2006, A\&A, 449, 451

Brandenburg, A. 2005, ApJ, 625, 539

Brassard, P., \& Fontaine, G. 1994, in IAU Colloq. 147: The

Equation of State in Astrophysics, ed. G. Chabrier \& E. Schatzman, 560

Brown, B. P., Miesch, M. S., Browning, M. K., Brun, A. S., \& Toomre, J. 2011, ApJ, 731, 69

Browning, M. K. 2008, ApJ, 676, 1262

Browning, M. K., Brun, A. S., \& Toomre, J. 2004, ApJ, 601, 512

Brun, A. S., Browning, M. K., Dikpati, M., Hotta, H., \& Strugarek, A. 2013, Space Sci. Rev., doi:10.1007/s11214-013-0028-0

Bullard, E., \& Gellman, H. 1954, Royal Society of London Philosophical Transactions Series A, 247, 213

Caligari, P., Moreno-Insertis, F., \& Schussler, M. 1995, ApJ, 441, 886

Caligari, P., Schüssler, M., \& Moreno-Insertis, F. 1998, ApJ, 502, 481

Cattaneo, F. 1999, ApJ, 515, L39

Cattaneo, F., Brummell, N. H., \& Cline, K. S. 2006, MNRAS, 365,727

Cattaneo, F., \& Hughes, D. W. 1988, Journal of Fluid Mechanics, 196,323

- 2006, Journal of Fluid Mechanics, 553, 401

Chabrier, G., \& Baraffe, I. 1997, A\&A, 327, 1039

Chabrier, G., Baraffe, I., Allard, F., \& Hauschildt, P. 2000, ApJ, 542,464

Chabrier, G., Gallardo, J., \& Baraffe, I. 2007, A\&A, 472, L17

Cheung, M. C. M., \& Isobe, H. 2014, Living Reviews in Solar Physics, 11, 3

Cheung, M. C. M., Moreno-Insertis, F., \& Schüssler, M. 2006, A\&A, 451, 303

Childress, S., \& Gilbert, A. D. 1995, Stretch, Twist, Fold

Choudhuri, A. R. 1989, Sol. Phys., 123, 217

Choudhuri, A. R., \& Gilman, P. A. 1987, ApJ, 316, 788

Cline, K. S., Brummell, N. H., \& Cattaneo, F. 2003, ApJ, 599, 1449

Davidson, P. A. 2013, Geophysical Journal International, 195, 67

Defouw, R. J. 1976, ApJ, 209, 266

DeLuca, E. E., Fan, Y., \& Saar, S. H. 1997, ApJ, 481, 369

Donati, J., Forveille, T., Cameron, A. C., et al. 2006, Science, 311,633

D'Silva, S., \& Choudhuri, A. R. 1993, A\&A, 272, 621

Fan, Y. 2001, Astrophys. J., 546, 509

Fan, Y. 2009, Living Reviews in Solar Physics, 6, 4

Fan, Y., Fisher, G. H., \& Deluca, E. E. 1993, ApJ, 405, 390

Favier, B., Jouve, L., Edmunds, W., Silvers, L. J., \& Proctor, M. R. E. 2012, MNRAS, 426, 3349

Featherstone, N. A., Browning, M. K., Brun, A. S., \& Toomre, J. 2009, ApJ, 705, 1000

Feiden, G. A., \& Chaboyer, B. 2012, ApJ, 761, 30

-. 2013, ApJ, 779, 183

-. 2014, ApJ, 789, 53

Finn, J. M., \& Ott, E. 1988, Physics of Fluids, 31, 2992

Gilman, P. A. 1970a, ApJ, 162, 1019

-. 1970b, ApJ, 162, 1019

Goldreich, P., \& Sridhar, S. 1995, ApJ, 438, 763

Gough, D. O., \& Tayler, R. J. 1966, MNRAS, 133, 85

Grappin, R., Frisch, U., Pouquet, A., \& Leorat, J. 1982, A\&A, 105,6

Hansen, C. J., \& Kawaler, S. D. 1994, Stellar Interiors. Physical Principles, Structure, and Evolution. (Springer-Verlag)

Hewitt, J. M., McKenzie, D. P., \& Weiss, N. O. 1975, Journal of Fluid Mechanics, 68, 721
Huang, X., \& Cumming, A. 2012, ApJ, 757, 47

Hubbard, W. B., \& Lampe, M. 1969, ApJS, 18, 297

Hughes, D. W. 1985, Geophysical and Astrophysical Fluid Dynamics, 34, 99

Hughes, D. W. 2007, in The Solar Tachocline, ed. D. W. Hughes, R. Rosner, \& N. O. Weiss, 275

Hughes, D. W., \& Cattaneo, F. 1987, Geophysical and Astrophysical Fluid Dynamics, 39, 65

Hughes, D. W., \& Falle, S. A. E. G. 1998, ApJ, 509, L57

Hughes, D. W., \& Proctor, M. R. E. 1988, Annual Review of Fluid Mechanics, 20, 187

Irwin, J. M., Quinn, S. N., Berta, Z. K., et al. 2011, ApJ, 742, 123

Itoh, N., Mitake, S., Iyetomi, H., \& Ichimaru, S. 1983, ApJ, 273, 774

Johns-Krull, C. M., \& Valenti, J. A. 1996, ApJ, 459, L95

Jones, C. 2008, in Les Houches, Vol. 88, Dynamos : Les Houches

Summer School, ed. Ph. Cardin and L.F. Cugliandolo, 45-135

Jones, C. A., \& Kuzanyan, K. M. 2009, Icarus, 204, 227

Jouve, L., \& Brun, A. S. 2009, ApJ, 701, 1300

Kersalé, E., Hughes, D. W., \& Tobias, S. M. 2007, ApJ, 663, L113

Klapper, I., \& Young, L. S. 1995, Communications in

Mathematical Physics, 173, 623

Kosovichev, A. G. 2002, Astronomische Nachrichten, 323, 186

Kosovichev, A. G., \& Duvall, T. L. 2006, Space Sci. Rev., 124, 1

Kraichnan, R. H. 1965, Physics of Fluids, 8, 1385

Kulsrud, R. M. 2005, Plasma physics for astrophysics (Princeton University Press)

Kulsrud, R. M., Cen, R., Ostriker, J. P., \& Ryu, D. 1997, ApJ, 480,481

Lazarian, A., \& Vishniac, E. T. 1999, ApJ, 517, 700

Liu, J., Goldreich, P. M., \& Stevenson, D. J. 2008, Icarus, 196, 653

López-Morales, M. 2007, ApJ, 660, 732

MacDonald, J., \& Mullan, D. J. 2012, MNRAS, 421, 3084

-. 2013, ApJ, 765, 126

-. 2014, ApJ, 787, 70

- 2015, MNRAS, 448, 2019

MacGregor, K. B., \& Cassinelli, J. P. 2003, ApJ, 586, 480

Martinez-Sykora, J., Moreno-Insertis, F., \& Cheung, M. C. M. 2015, ArXiv e-prints

Matsumoto, R., \& Shibata, K. 1992, Publ. Astron. Soc. Japan, 44, 167

Matthews, P. C., Hughes, D. W., \& Proctor, M. R. E. 1995, ApJ, 448, 938

McQuillan, A., Mazeh, T., \& Aigrain, S. 2014, ApJS, 211, 24

Meakin, C. A., \& Arnett, D. 2007, ApJ, 667, 448

Miesch, M. S. 2005, Living Reviews in Solar Physics, 2, 1

Mitake, S., Ichimaru, S., \& Itoh, N. 1984, ApJ, 277, 375

Moffatt, H. K. 1978, Magnetic field generation in electrically conducting fluids (Cambridge, England, Cambridge University Press, 1978. 353 p.)

Morales, J. C., Ribas, I., \& Jordi, C. 2008, A\&A, 478, 507

Moreno-Insertis, F. 1983, A\&A, 122, 241

- .1986, A\&A, 166, 291

Moreno-Insertis, F., Caligari, P., \& Schuessler, M. 1995, ApJ, 452, 894

Mullan, D. J., \& MacDonald, J. 2001, ApJ, 559, 353

Nelson, N. J., Brown, B. P., Brun, A. S., Miesch, M. S., \& Toomre, J. 2011, ApJ, 739, L38

Nelson, N. J., Brown, B. P., Sacha Brun, A., Miesch, M. S., \& Toomre, J. 2014, Sol. Phys., 289, 441

Newcomb, W. A. 1961, Physics of Fluids, 4, 391

Noyes, R. W., Hartmann, L. W., Baliunas, S. L., Duncan, D. K., \& Vaughan, A. H. 1984, ApJ, 279, 763

Nozawa, S. 2005, PASJ, 57, 995

Oruba, L., \& Dormy, E. 2014, Geophysical Journal International, 198,828

Ossendrijver, M. 2003, A\&A Rev., 11, 287

Osten, R. A., Godet, O., Drake, S., \& al. 2010, ApJ, 721, 785

Parker, E. N. 1955, ApJ, 121, 491

-. 1966, ApJ, 145, 811

-. 1975, ApJ, 198, 205

-. 1984, ApJ, 283, 343

-. 1993, ApJ, 408, 707

Passot, T., Vazquez-Semadeni, E., \& Pouquet, A. 1995, ApJ, 455, 536 
Perez, J. C., Mason, J., Boldyrev, S., \& Cattaneo, F. 2014, ApJ, 793, L13

Pinto, R. F., \& Brun, A. S. 2013, ApJ, 772, 55

Pizzolato, N., Maggio, A., Micela, G., Sciortino, S., \& Ventura, P. 2003, A\&A, 397, 147

Potekhin, A. Y. 1999, A\&A, 351, 787

Potekhin, A. Y., Baiko, D. A., Haensel, P., \& Yakovlev, D. G. 1999, A\&A, 346, 345

Rauscher, E., \& Menou, K. 2013, ApJ, 764, 103

Reiners, A., \& Basri, G. 2007, ApJ, 656, 1121

Roberts, B., \& Webb, A. R. 1978, Sol. Phys., 56, 5

Roberts, P., Jones, C., \& Calderwood, A. 2003, Energy fluxes and ohmic dissipation in the earth's core, ed. K. Zhang, A. Soward, \& C. Jones, 100-129

Roberts, P. H. 2009, in IAU Symposium, Vol. 259, IAU Symposium, ed. K. G. Strassmeier, A. G. Kosovichev, \& J. E. Beckman, 259-270

Roberts, P. H., \& King, E. M. 2013, Reports on Progress in Physics, 76, 096801

Rogers, T. M., \& Komacek, T. D. 2014, ApJ, 794, 132

Rogers, T. M., \& Showman, A. P. 2014, ApJ, 782, L4

Schmitt, J. H. M. M., \& Rosner, R. 1983, ApJ, 265, 901

Schuessler, M. 1977, A\&A, 56, 439

Schüssler, M., Caligari, P., Ferriz-Mas, A., Solanki, S. K., \& Stix, M. 1996, A\&A, 314, 503

Schüssler, M., \& Solanki, S. K. 1992, A\&A, 264, L13

Shibata, K., Tajima, T., Matsumoto, R., et al. 1989a, Astrophys. J., 338, 471

Shibata, K., Tajima, T., Steinolfson, R. S., \& Matsumoto, R. 1989b, Astrophys. J., 345, 584

Spruit, H. C. 1981a, A\&A, 102, 129

-. 1981b, A\&A, 98, 155

-. 2002, A\&A, 381, 923

Spruit, H. C., \& van Ballegooijen, A. A. 1982, A\&A, 106, 58
Spruit, H. C., \& Weiss, A. 1986, A\&A, 166, 167

Sridhar, S., \& Goldreich, P. 1994, ApJ, 432, 612

Stassun, K. G., Kratter, K. M., Scholz, A., \& Dupuy, T. J. 2012, ApJ, 756, 47

Stelzer, Z., \& Jackson, A. 2013, Geophysical Journal International, 193, 1265

Thomas, J. H., \& Nye, A. H. 1975, Physics of Fluids, 18, 490

Thompson, C., \& Duncan, R. C. 1993, ApJ, 408, 194

Thompson, M. J., Toomre, J., Anderson, E. R., et al. 1996, Science, 272, 1300

Tobias, S. M., Brummell, N. H., Clune, T. L., \& Toomre, J. 1998, ApJ, 502, L177

-. 2001, ApJ, 549, 1183

Tobias, S. M., Cattaneo, F., \& Brummell, N. H. 2008, ApJ, 685 , 596

Tobias, S. M., \& Hughes, D. W. 2004, ApJ, 603, 785

Torres, G. 2013, Astronomische Nachrichten, 334, 4

Torres, G., \& Ribas, I. 2002, ApJ, 567, 1140

Unno, W., \& Ribes, E. 1976, ApJ, 208, 222

Vasil, G. M., \& Brummell, N. H. 2009, ApJ, 690, 783

Viallet, M., Meakin, C., Arnett, D., \& Mocák, M. 2013, ApJ, 769, 1

Weber, M. A., Fan, Y., \& Miesch, M. S. 2011, ApJ, 741, 11

Weiss, N. O., Thomas, J. H., Brummell, N. H., \& Tobias, S. M. 2004, ApJ, 600, 1073

Wissink, J. G., Matthews, P. C., Hughes, D. W., \& Proctor,

M. R. E. 2000, ApJ, 536, 982

Wright, N. J., Drake, J. J., Mamajek, E. E., \& Henry, G. W. 2011, ApJ, 743, 48

Wu, Y., \& Lithwick, Y. 2013, ApJ, 763, 13

Zahn, J.-P., Brun, A. S., \& Mathis, S. 2007, A\&A, 474, 145 